\title{
Fracture Analysis of Bounded Magnetoelectroelastic Layers with Interfacial Cracks under Magnetoelectromechanical Loads: Plane Problem
}

\author{
W. J. Fenga, R.K.L. Su, ${ }^{b, *}$ J. X. Liu', Y. S. Li \\ ${ }^{a}$ Department of Engineering Mechanics, Shijiazhuang Railway Institute, Shijiazhuang 050043, P. R. China \\ ${ }^{\mathrm{b}}$ Department of Civil Engineering, The University of Hong Kong, P. R. China \\ 'Institute of Engineering Mechanics, Northeastern University, Shenyang 110004, P. R. China
}

\begin{abstract}
Fracture behaviors of multiple interfacial cracks between dissimilar magnetoelectroelastic layers subjected to in-plane magnetoelectromechanical loads are investigated by using integral transform method and singular integral equation technique. The number of the interfacial cracks is arbitrary, and the crack surfaces are assumed to be magnetoelectrically impermeable. The field intensity factors (FIFs) including stress, electric displacement and magnetic induction intensity factors as well as the energy release rates (ERRs) are derived. The effects of loading combinations, crack configurations and material property parameters on the fracture behaviors are evaluated according to energy release rate criterion. Numerical results show that both negative electrical and magnetic loads inhibit crack extension, and that the material constants have different and important effects on the ERRs. The results presented here should have potential applications to the design of multilayered magnetoelectroelastic structures.
\end{abstract}

Keywords: Mode-I problem; Magnetoelectroelastic layer; Multiple interfacial cracks;

\footnotetext{
* Corresponding author. Tel: 852 28592648; Fax: 85225595337

E-mail address: klsu@hkucc.hku.hk (R.K.L. Su)
} 
Energy release rate; Singular integral equation

\section{Introduction}

Magnetoelectroelastic materials have been widely used in electronics industry. The technical applications may include waveguides, sensors, phase invertors, transducers, etc. (Parton and Kudryavtsev, 1988). Studies on the properties of these kinds of composites have been carried out in recent years (Harshe et al., 1993; Nan, 1994; Alshits et al., 1995; Huang and Kuo, 1997; Huang et al. 1998; Li and Dunn, 1998; Li, 2000; Wang and Shen, 2003).

In the design of magnetoelectroelastic structures, it is important to take into account imperfections, such as cracks including multiple cracks, which are often pre-existing or are generated by external loads during the service life. Therefore, research on fracture mechanics of magnetoelectroelastic materials has also drawn increased interests (Zhou etal., 2004; Gao et al., 2004; Chue and Liu, 2005; Feng et al., 2005; Li, 2005; Feng and Su, 2006; Li and Kardomateas, 2006; Wang et al., 2006; Zhao et al., 2006; Feng et al., 2007; Yong and Zhou, 2007; Wang et al., 2008)

For two dimensional (2-D) plane crack problems, Liu et al. (2001) derived the Green's functions for an infinite magnetoelectroelastic plane containing an elliptic cavity. They reduced the cavity solution to obtain the solution for a permeable crack. Gao et al. (2003a, b) analyzed single and collinear cracks in an infinite magnetoelectroelastic material and obtained the extended stress intensity factors. Song and Sih (2003) and Sih et al. (2003) investigated the influence of both magnetic field and electric field on crack growth, in particular, on crack initiation angle under various crack surface conditions for mode-I, mode-II, and mixed mode crack models. Tian and Gabbert (2004) and Tian and Rajapakse (2005) studied the interaction problem of multiple arbitrarily oriented and distributed cracks in homogeneous 
magnetoelectroelastic materials. Wang and Mai (2007) discussed the effects of four kinds of ideally magnetoelectric crack-face conditions on fracture properties of magnetoelectroelastic materials. Zhong and Li (2007) obtained the T-stress for a Griffith crack in an infinite magnetoelectroelastic medium based on magnetic and electric boundary conditions nonlinearly dependent on the crack opening displacement. Zhou et al. (2007; 2008) investigated the static fracture behaviors of a single crack or two cracks in piezoelectric/piezomagnetic materials by the Schmidt method. However, all the above-mentioned works are related to crack in a homogenous magnetoelectroelastic medium. Due to the oscillating singularity of crack tips, the study of interfacial crack between dissimilar magnetoelectroelastic materials is very limited. Gao et al. (2003c) derived the exact solution for a permeable interfacial crack between two dissimilar magnetoelectroelastic solids under general applied loads. Li and Kardomateas (2007) investigated the interfacial crack problem of dissimilar piezoelectromagneto-elastic anisotropic bimaterials under in-plane deformation taking the electric-magnetic field inside the interfacial crack into account. Up till now, to the best of our knowledge, the fracture problems of multiple interfacial cracks between dissimilar unbounded magnetoelectroelastic materials have not yet been reported, let alone for the problems of interfacial cracks between two finite magnetoelectroelastic layers.

In this paper, fracture analyses of multiple collinear interfacial cracks between dissimilar magnetoelectroelastic layers are conducted. The magnetoelectrically impermeable crack surface condition is adopted. The field intensity factors (FIFs) are derived by using the integral transform and singular integral equation methods. The energy release rates (ERRs) are further obtained and numerically solved. The effects of applied magnetoelectromechanical loads, layer heights especially material 
combination parameters on the fracture behaviors are discussed in detail. The results could be of particular interest to the analysis and design of smart sensors/actuators constructed from magnetoelectroelastic composite laminates.

\section{Formulation of the problem}

Consider $n$ cracks along the interface between two transversely isotropic magnetroelectroelastic layers with both poling directions as the $z$-axis as shown in Fig. 1. The $k$ th crack lies from $a_{k}$ to $b_{k}(k=1 \sim n)$.

For the 2-D plane strain problem considered here, the constitutive equations within the framework of the theory of linear magnetoelectroelastic medium take the form (Huang and Kuo, 1997)

$$
\begin{aligned}
& \left\{\begin{array}{l}
\sigma_{x x} \\
\sigma_{z z} \\
\sigma_{x z}
\end{array}\right\}=\left[\begin{array}{ccc}
c_{11} & c_{13} & 0 \\
c_{13} & c_{33} & 0 \\
0 & 0 & c_{44}
\end{array}\right]\left\{\begin{array}{c}
u_{, x} \\
w_{, z} \\
u_{, z}+w_{, x}
\end{array}\right\}+\left[\begin{array}{cc}
0 & e_{13} \\
0 & e_{33} \\
e_{15} & 0
\end{array}\right]\left\{\begin{array}{l}
\phi_{, x} \\
\phi_{, z}
\end{array}\right\}+\left[\begin{array}{cc}
0 & f_{13} \\
0 & f_{33} \\
f_{15} & 0
\end{array}\right]\left\{\begin{array}{l}
\psi_{, x} \\
\psi_{, z}
\end{array}\right\}, \\
& \left\{\begin{array}{l}
D_{x} \\
D_{z}
\end{array}\right\}=\left[\begin{array}{ccc}
0 & 0 & e_{15} \\
e_{13} & e_{33} & 0
\end{array}\right]\left\{\begin{array}{c}
u_{, x} \\
w_{, z} \\
u_{, z}+w_{, x}
\end{array}\right\}-\left[\begin{array}{cc}
\varepsilon_{11} & 0 \\
0 & \varepsilon_{33}
\end{array}\right]\left\{\begin{array}{l}
\phi_{, x} \\
\phi_{, z}
\end{array}\right\}-\left[\begin{array}{cc}
g_{11} & 0 \\
0 & g_{33}
\end{array}\right]\left\{\begin{array}{l}
\psi_{, x} \\
\psi_{, z}
\end{array}\right\}, \\
& \left\{\begin{array}{l}
B_{x} \\
B_{z}
\end{array}\right\}=\left[\begin{array}{ccc}
0 & 0 & f_{15} \\
f_{31} & f_{33} & 0
\end{array}\right]\left\{\begin{array}{c}
u_{, x} \\
w_{, z} \\
u_{, z}+w_{, x}
\end{array}\right\}-\left[\begin{array}{cc}
g_{11} & 0 \\
0 & g_{33}
\end{array}\right]\left\{\begin{array}{l}
\phi_{, x} \\
\phi_{, z}
\end{array}\right\}-\left[\begin{array}{cc}
\mu_{11} & 0 \\
0 & \mu_{33}
\end{array}\right]\left\{\begin{array}{l}
\psi_{, x} \\
\psi_{, z}
\end{array}\right\},
\end{aligned}
$$

where $u$ and $w$ are the displacement components; $\phi$ and $\psi$ are the electric and magnetic potentials, respectively; $\sigma_{i j}, D_{i}$ and $B_{i} \quad(i, j=x, z)$ are the stresses, electric displacements and magnetic inductions, respectively; $c_{i j}, e_{i j}, f_{i j}$ and $g_{i j}$ $(i, j=1,3)$ are the elastic, piezoelectric, piezomagnetic and magnetoelectric constants, respectively; $\varepsilon_{i j}$ and $\mu_{i j} \quad(i, j=1,3)$ are the dielectric permitivities and magnetic permitivities, respectively.

In the absence of body forces, free charges and electric charge density, the 
governing equations for elastic displacements $u$ and $w$, electric potential $\phi$, and magnetic potential $\psi$ can be written as follows

$$
\begin{aligned}
& c_{11} u_{, x x}+c_{44} u_{, z z}+\left(c_{13}+c_{44}\right) w_{, x z}+\left(e_{13}+e_{15}\right) \phi_{, x z}+\left(f_{13}+f_{15}\right) \psi_{, x z}=0, \\
& \left(c_{13}+c_{44}\right) u_{, x z}+c_{44} w_{, x x}+c_{33} w_{, z z}+e_{15} \phi_{, x x}+e_{33} \phi_{, z z}+f_{15} \psi_{, x x}+f_{33} \psi_{, z z}=0, \\
& \left(e_{13}+e_{15}\right) u_{, x z}+e_{15} w_{, x x}+e_{33} w_{, z z}-\varepsilon_{11} \phi_{, x x}-\varepsilon_{33} \phi_{, z z}-g_{11} \psi_{, x x}-g_{33} \psi_{, z z}=0, \\
& \left(f_{13}+f_{15}\right) u_{, x z}+f_{15} w_{, x x}+f_{33} w_{, z z}-g_{11} \phi_{, x x}-g_{33} \phi_{, z z}-\mu_{11} \psi_{, x x}-\mu_{33} \psi_{, z z}=0 .
\end{aligned}
$$

For the magnetoelectrically impermeable interfacial cracks considered in this study, the boundary and continuity conditions are

$$
\begin{aligned}
& \sigma_{x z}^{(1)}(x, 0)=\sigma_{x z}^{(2)}(x, 0)=-\tau_{0}, \quad x \in \bigcup_{k=1}^{n}\left(a_{k}, b_{k}\right), \\
& \sigma_{z z}^{(1)}(x, 0)=\sigma_{z z}^{(2)}(x, 0)=-\sigma_{0}, \quad x \in \bigcup_{k=1}^{n}\left(a_{k}, b_{k}\right), \\
& D_{z}^{(1)}(x, 0)=D_{z}^{(2)}(x, 0)=-D_{0}, \quad x \in \bigcup_{k=1}^{n}\left(a_{k}, b_{k}\right), \\
& B_{z}^{(1)}(x, 0)=B_{z}^{(2)}(x, 0)=-B_{0}, \quad x \in \bigcup_{k=1}^{n}\left(a_{k}, b_{k}\right), \\
& u^{(1)}(x, 0)-u^{(2)}(x, 0)=\Delta u(x), \quad w^{(1)}(x, 0)-w^{(2)}(x, 0)=\Delta w(x), \quad-\infty<x<+\infty, \\
& \phi^{(1)}(x, 0)-\phi^{(2)}(x, 0)=\Delta \phi(x), \quad \varphi^{(1)}(x, 0)-\varphi^{(2)}(x, 0)=\Delta \varphi(x), \quad-\infty<x<+\infty, \\
& \sigma_{x z}^{(1)}(x, 0)=\sigma_{x z}^{(2)}(x, 0), \quad \sigma_{z z}^{(1)}(x, 0)=\sigma_{z z}^{(2)}(x, 0), \quad-\infty<x<+\infty, \\
& D_{z}^{(1)}(x, 0)=D_{z}^{(2)}(x, 0), \quad B_{z}^{(1)}(x, 0)=B_{z}^{(2)}(x, 0), \quad-\infty<x<+\infty, \\
& \sigma_{x z}^{(1)}\left(x, h_{1}\right)=0, \quad \sigma_{z z}^{(1)}\left(x, h_{1}\right)=0, \quad-\infty<x<+\infty, \\
& D_{z}^{(1)}\left(x, h_{1}\right)=0, \quad B_{z}^{(1)}\left(x, h_{1}\right)=0, \quad-\infty<x<+\infty, \\
& \sigma_{x z}^{(2)}\left(x,-h_{2}\right)=0, \quad \sigma_{z z}^{(2)}\left(x,-h_{2}\right)=0, \quad-\infty<x<+\infty, \\
& D_{z}^{(2)}\left(x,-h_{2}\right)=0, \quad B_{z}^{(2)}\left(x,-h_{2}\right)=0, \quad-\infty<x<+\infty,
\end{aligned}
$$

where the superscripts (1) and (2) denote mediums 1 and 2, respectively; $\tau_{0}, \sigma_{0}, D_{0}$ 
and $B_{0}$ are respectively the given shear stress, normal stress, electric displacement and magnetic induction applied on the crack-faces; $h_{1}$ and $h_{2}$ are the heights of mediums 1 and 2, respectively; $\Delta u, \Delta w, \Delta \phi$ and $\Delta \varphi$ are introduced extended displacement jump functions, i.e.,

$$
\begin{aligned}
& \Delta u(x)= \begin{cases}\Delta u_{k}(x)=u^{(1)}(x, 0)-u^{(2)}(x, 0), & x \in\left(a_{k}, b_{k}\right), k=1,2, \cdots, n, \\
0, & x \notin \bigcup_{k=1}^{n}\left(a_{k}, b_{k}\right),\end{cases} \\
& \Delta w(x)= \begin{cases}\Delta w_{k}(x)=w^{(1)}(x, 0)-w^{(2)}(x, 0), & x \in\left(a_{k}, b_{k}\right), k=1,2, \cdots, n, \\
0, & x \notin \bigcup_{k=1}^{n}\left(a_{k}, b_{k}\right),\end{cases} \\
& \Delta \phi(x)= \begin{cases}\Delta \phi_{k}(x)=\phi^{(1)}(x, 0)-\phi^{(2)}(x, 0), & x \in\left(a_{k}, b_{k}\right), k=1,2, \cdots, n, \\
0, & x \notin \bigcup_{k=1}^{n}\left(a_{k}, b_{k}\right),\end{cases} \\
& \Delta \varphi(x)= \begin{cases}\Delta \varphi_{k}(x)=\varphi^{(1)}(x, 0)-\varphi^{(2)}(x, 0), & x \in\left(a_{k}, b_{k}\right), k=1,2, \cdots, n, \\
0, & x \notin \bigcup_{k=1}^{n}\left(a_{k}, b_{k}\right) .\end{cases}
\end{aligned}
$$

\section{Derivation and solutions of singular integral equations}

For solving crack problems, two kinds of methods are usually applied. They are the Fourier transform method (including singular integral equation technique) and the complex variable method. To the best of our knowledge, the complex variable method is not able to be used to solve crack problems of finite body, let alone interfacial crack problems of bonded magnetroelectroelastic layers. On the other hand, the Fourier transform technique has been widely used to solve BVP of piezoelectric ceramics (Soh et al. 2000; Gu et al., 2002). In this paper, we intend to extend the work of Gu et al. (2002) for piezoelectric bimaterials to magnetoelectroelastic bimaterials with multiple interfacial cracks. 
We define a Fourier transform pair as follows:

$$
\begin{aligned}
& \bar{U}(s, z)=\int_{-\infty}^{\infty} U(x, z) e^{-i s x} d x, \\
& U(x, z)=\frac{1}{2 \pi} \int_{-\infty}^{\infty} \bar{U}(s, z) e^{i s x} d s,
\end{aligned}
$$

where $\bar{U}(s, z)$ and $U(s, z)$ are respectively the function in the Fourier transform domain and the original one; $\mathrm{s}$ is the Fourier transform parameter.

Appling Fourier transforms to Eqs. (2), we can obtain

$$
\begin{aligned}
& -s^{2} c_{11} \bar{u}+c_{44} \bar{u}_{, z z}+(i s)\left(c_{13}+c_{44}\right) \bar{w}_{, z}+(i s)\left(e_{13}+e_{15}\right) \bar{\phi}_{, z}+(i s)\left(f_{13}+f_{15}\right) \bar{\psi}_{, z}=0, \\
& (i s)\left(c_{13}+c_{44}\right) \bar{u}_{, z}-s^{2} c_{44} \bar{w}+c_{33} \bar{w}_{, z z}-s^{2} e_{15} \bar{\phi}+e_{33} \bar{\phi}_{, z z}-s^{2} f_{15} \bar{\psi}+f_{33} \bar{\psi}_{, z z}=0, \\
& (i s)\left(e_{13}+e_{15}\right) \bar{u}_{, z}-s^{2} e_{15} \bar{w}+e_{33} \bar{w}_{, z z}+s^{2} \varepsilon_{11} \bar{\phi}-\varepsilon_{33} \bar{\phi}_{, z z}+s^{2} g_{11} \bar{\psi}-g_{33} \bar{\psi}_{, z z}=0, \\
& (i s)\left(f_{13}+f_{15}\right) \bar{u}_{, z}-s^{2} f_{15} \bar{w}+f_{33} \bar{w}_{, z z}+s^{2} g_{11} \bar{\phi}-g_{33} \bar{\phi}_{, z}+s^{2} \mu_{11} \bar{\psi}-\mu_{33} \bar{\psi}_{, z z}=0 .
\end{aligned}
$$

Eqs. (6) are second order system of ordinary differential equations, similar to the solutions in Soh et al. (2000) and/or in Gu et al. (2002), and the solutions of Eqs. (6) can be easily obtained. Thus, further applying inverse Fourier transforms, the elastic displacements, electric potentials and magnetic potentials in Eqs. (2) can be expressed as

$$
\begin{aligned}
& u^{(\alpha)}(x, z)=\frac{1}{2 \pi} \int_{-\infty}^{\infty}\left[\sum_{j=1}^{8} A_{j}^{(\alpha)}(s) e^{\lambda_{j}^{(\alpha)} z}\right] e^{-i s x} d s, \\
& w^{(\alpha)}(x, z)=\frac{1}{2 \pi} \int_{-\infty}^{\infty}\left[\sum_{j=1}^{8} a_{j}^{(\alpha)}(s) A_{j}^{(\alpha)}(s) e^{\lambda_{j}^{(\alpha)} z}\right] e^{-i s x} d s, \\
& \phi^{(\alpha)}(x, z)=\frac{1}{2 \pi} \int_{-\infty}^{\infty}\left[\sum_{j=1}^{8} b_{j}^{(\alpha)}(s) A_{j}^{(\alpha)}(s) e^{\lambda_{j}^{(\alpha)} z}\right] e^{-i s x} d s, \\
& \psi^{(\alpha)}(x, z)=\frac{1}{2 \pi} \int_{-\infty}^{\infty}\left[\sum_{j=1}^{8} c_{j}^{(\alpha)}(s) A_{j}^{(\alpha)}(s) e^{\lambda_{j}^{(\alpha)} z}\right] e^{-i s x} d s,
\end{aligned}
$$

where the superscript $\alpha(\alpha=1,2)$ stands for the corresponding medium. $a_{j}^{(\alpha)}(s)$, $b_{j}^{(\alpha)}(s), \quad c_{j}^{(\alpha)}(s)$ and $\lambda_{j}^{(\alpha)}(s)(j=1,2, \cdots \cdots, 8)$ are some known functions of the Fourier 
variety $s$ (see Appendix A), and the parameters $A_{j}^{(\alpha)}(s)(j=1,2, \cdots \cdots, 8)$ are yet unknown.

Substituting Eqs. (7) into Eqs. (4) and using Eqs. (3e)-(3l), we have

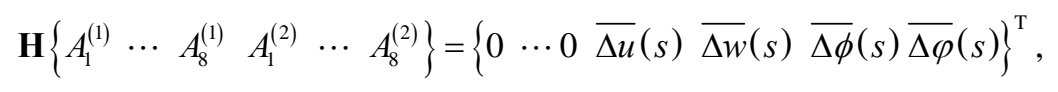

where $\mathbf{H}$ is a $16 \times 16$ matrix, the elements of which are given in Appendix B. $\overline{\Delta u}(s)$, $\overline{\Delta w}(s), \overline{\Delta \phi}(s)$ and $\overline{\Delta \psi}(s)$ are the Fourier transforms of $\Delta u(x), \Delta w(x), \Delta \phi(s)$ and $\Delta \psi(x)$, respectively.

According to the Cramer's rule, we get from Eq. (8)

$$
A_{i}^{(1)}=\frac{\Delta_{13 i}(s) \overline{\Delta u}(s)+\Delta_{14 i}(s, p) \overline{\Delta w}(s)+\Delta_{15 i}(s) \overline{\Delta \phi}(s)+\Delta_{16 i}(s, p) \overline{\Delta \varphi}(s)}{\Delta(s)}, \quad i=1,2, \cdots, 8,
$$

where $\Delta(s)$ is the determinant of the coefficient matrix of Eq. (8), $\Delta_{13 i}(s), \Delta_{14 i}(s)$ $\Delta_{15 i}(s)$ and $\Delta_{16 i}(s)$ are respectively the corresponding algebra cofactors.

Substituting Eqs. (7) into Eqs. (1) and using Eqs. (3a)-(3d) and Eq. (9), we have

$$
\frac{1}{2 \pi} \int_{-\infty}^{+\infty} \mathbf{P}(s) \overline{\mathbf{V}}(s) e^{-i s x} d s=\boldsymbol{\Gamma}(x), \quad x \in \bigcup_{k=1}^{n}\left(a_{k}, b_{k}\right),
$$

where

$$
\begin{aligned}
& \mathbf{P}(s)=\left[\begin{array}{llll}
\sum_{j=1}^{8} \frac{h_{10 j} \Delta_{13 j}(s)}{\Delta(s)} & \sum_{j=1}^{8} \frac{h_{10 j} \Delta_{14 j}(s)}{\Delta(s)} & \sum_{j=1}^{8} \frac{h_{10 j} \Delta_{15 j}(s)}{\Delta(s)} & \sum_{j=1}^{8} \frac{h_{10 j} \Delta_{16 j}(s)}{\Delta(s)} \\
\sum_{j=1}^{8} \frac{h_{9 j} \Delta_{13 j}(s)}{\Delta(s)} & \sum_{j=1}^{8} \frac{h_{9 j} \Delta_{14 j}(s)}{\Delta(s)} & \sum_{j=1}^{8} \frac{h_{9 j} \Delta_{15 j}(s)}{\Delta(s)} & \sum_{j=1}^{8} \frac{h_{9 j} \Delta_{16 j}(s)}{\Delta(s)} \\
\sum_{j=1}^{8} \frac{h_{11 j} \Delta_{13 j}(s)}{\Delta(s)} & \sum_{j=1}^{8} \frac{h_{11 j} \Delta_{14 j}(s)}{\Delta(s)} & \sum_{j=1}^{8} \frac{h_{11 j} \Delta_{15 j}(s)}{\Delta(s)} & \sum_{j=1}^{8} \frac{h_{11 j} \Delta_{16 j}(s)}{\Delta(s)} \\
\sum_{j=1}^{8} \frac{h_{12 j} \Delta_{13 j}(s)}{\Delta(s)} & \sum_{j=1}^{8} \frac{h_{12 j} \Delta_{14 j}(s)}{\Delta(s)} & \sum_{j=1}^{8} \frac{h_{12 j} \Delta_{15 j}(s)}{\Delta(s)} & \sum_{j=1}^{8} \frac{h_{12 j} \Delta_{16 j}(s)}{\Delta(s)}
\end{array}\right], \\
& \overline{\mathbf{V}}(s)=\left\{\begin{array}{lllll}
\overline{\Delta u}(s) & \overline{\Delta w}(s) & \overline{\Delta \phi}(s) & \overline{\Delta \varphi}(s)
\end{array}\right\}^{\mathrm{T}}, \\
& \boldsymbol{\Gamma}(x)=\left\{\begin{array}{llll}
-\tau_{0}(x) & -\sigma_{0}(x) & -D_{0}(x) & -B_{0}(x)
\end{array}\right\}^{\mathrm{T}} .
\end{aligned}
$$

Applying inverse Fourier transform for $\overline{\mathbf{V}}(s)$, we get the following equation from 
Eq.(10) (Su et al., 2003)

$$
\frac{1}{2 \pi} \int_{-\infty}^{+\infty} \sum_{k=1}^{n}\left[\int_{a_{k}}^{b_{k}} \mathbf{P}(s) \mathbf{V}_{k}(v) e^{i s(v-x)} d v\right] d s=\boldsymbol{\Gamma}(x), \quad x \in \bigcup_{l=1}^{n}\left(a_{l}, b_{l}\right)
$$

where

$$
\mathbf{V}_{k}(v)=\left\{\Delta u_{k}(v) \quad \Delta w_{k}(v) \quad \Delta \phi_{k}(v) \quad \Delta \varphi_{k}(v)\right\}^{\mathrm{T}}
$$

By partial integration and introducing dislocation density functions of the $k$ th crack (Su et al., 2003)

$$
\begin{aligned}
\mathbf{F}_{k}(v) & =\left\{\begin{array}{llll}
f_{1}(v) & f_{2}(v) & f_{3}(v) & f_{4}(v)
\end{array}\right\}^{\mathrm{T}} \\
& =\left\{\begin{array}{llll}
\frac{\partial \Delta u_{k}(v)}{\partial v} & \frac{\partial \Delta w_{k}(v)}{\partial v} & \frac{\partial \Delta \phi_{k}(v)}{\partial v} & \frac{\partial \Delta \psi_{k}(v)}{\partial v}
\end{array}\right\}^{\mathrm{T}}, k=1,2, \cdots n,
\end{aligned}
$$

we can easily obtain from Eq. (12)

$$
\frac{1}{2 \pi} \int_{-\infty}^{+\infty} \sum_{k=1}^{n}\left[\int_{a_{k}}^{b_{k}} \frac{\mathbf{P}(s)}{(-i s)} \mathbf{F}_{k}(v) e^{i s(v-x)} d v\right] d s=\boldsymbol{\Gamma}(x), \quad x \in \bigcup_{l=1}^{n}\left(a_{l}, b_{l}\right)
$$

By exchanging the integral order, Eq. (15) can be further transformed into the following form (Su et al., 2003)

$$
\frac{1}{2 \pi} \sum_{k=1}^{n} \int_{a_{k}}^{b_{k}} \int_{-\infty}^{+\infty} \frac{\mathbf{P}(s)}{(-i s)} \mathbf{F}_{k}(v) e^{i s(v-x)} d s d v=\boldsymbol{\Gamma}(x), \quad x \in \bigcup_{l=1}^{n}\left(a_{l}, b_{l}\right) .
$$

It is clear that the singularities of the integral equations are attributable to the asymptotic value of matrix $\mathrm{P}$ as $|s| \rightarrow \infty$. Similar to the case of piezoelectric media solved by Gu et al. (2002), we get

$$
\mathbf{A F}_{l}(x)+\frac{1}{\pi} \sum_{k=1}^{n} \int_{a_{k}}^{b_{k}} \mathbf{B} \frac{\mathbf{F}_{k}(v)}{v-x} d v+\frac{1}{\pi} \sum_{k=1}^{n} \int_{a_{k}}^{b_{k}} \mathbf{Q}(v, x) \mathbf{F}_{k}(v) d v=\boldsymbol{\Gamma}(x), \quad x \in\left(a_{l}, b_{l}\right)
$$

where $\mathbf{A}$ and $\mathbf{B}$ are two known constant matrices with respect to the material constants in Eqs. (1), and $\mathbf{Q}(v, x)$ is a known function matrix (Appendix B). It should be noted that in the deriving of Eq. (17), the following relation is used

$$
\int_{0}^{\infty} \sin [s(v-x)] d s=\frac{1}{v-x}
$$


By introducing two non-dimensional variables $\eta$ and $\xi$, i.e.,

$$
v=\frac{b_{k}-a_{k}}{2} \eta+\frac{b_{k}+a_{k}}{2}, \quad x=\frac{b_{k}-a_{k}}{2} \xi+\frac{b_{k}+a_{k}}{2}, \quad v, x \in\left(a_{k}, b_{k}\right), k=1,2, \mathrm{~L}, n,
$$

We can obtain from Eq. (17)

$$
\mathbf{A} \tilde{\mathbf{F}}_{l}(\xi)+\frac{1}{\pi} \int_{-1}^{1} \mathbf{B} \frac{\tilde{\mathbf{F}}_{l}(\eta)}{\eta-\xi} d \eta+\sum_{k=1}^{n} \frac{1}{\pi} \int_{-1}^{1} \tilde{\mathbf{Q}}^{k l}(\eta, \xi) \tilde{\mathbf{F}}_{k}(\eta) d \eta=\tilde{\boldsymbol{\Gamma}}_{l}(\xi), \quad l=1,2, \cdots, n,
$$

where

$$
\begin{aligned}
& \tilde{\mathbf{F}}_{k}(\eta)=\mathbf{F}_{k}\left(\frac{b_{k}-a_{k}}{2} \eta+\frac{b_{k}+a_{k}}{2}\right), \\
& \tilde{\mathbf{Q}}^{k l}(\eta, \xi)=\frac{b_{k}-a_{k}}{2} \mathbf{Q}\left(\frac{b_{k}-a_{k}}{2} \eta+\frac{b_{k}+a_{k}}{2}, \frac{b_{l}-a_{l}}{2} \xi+\frac{b_{l}+a_{l}}{2}\right) \\
& \quad+\frac{b_{k}-a_{k}}{2}\left(1-\delta_{k l}\right) \mathbf{B} /\left[\left(\frac{b_{k}-a_{k}}{2} \eta+\frac{b_{k}+a_{k}}{2}\right)-\left(\frac{b_{l}-a_{l}}{2} \xi+\frac{b_{l}+a_{l}}{2}\right)\right], \\
& \tilde{\boldsymbol{\Gamma}}_{k}(\xi)=\boldsymbol{\Gamma}\left(\frac{b_{k}-a_{k}}{2} \xi+\frac{b_{k}+a_{k}}{2}\right) .
\end{aligned}
$$

An approximate method described by Shen and Kuang (1998) is employed to solve the Cauchy singular integral equation (20) of the second type. The method was also used by Gu et al. (2002) in solving a single interface crack problem of bounded piezoelectric layers. The detailed deriving process is as follows.

The regularization of Eq. (20) leads to

$$
\Lambda \boldsymbol{\psi}_{l}(\xi)+\frac{1}{\pi} \int_{-1}^{1} \frac{\boldsymbol{\Psi}_{l}(\eta)}{\eta-\xi} d \eta+\sum_{k=1}^{n} \frac{1}{\pi} \int_{-1}^{1} \overline{\mathbf{Q}}^{k l}(\eta, \xi) \boldsymbol{\Psi}_{k}(\eta) d \eta=\overline{\mathbf{L}}_{l}(\xi), \quad l=1,2, \cdots, n,
$$

where

$$
\boldsymbol{\Psi}_{k}(\eta)=\mathbf{R}^{-1} \tilde{\mathbf{F}}_{k}(\eta), \quad \overline{\mathbf{Q}}^{k l}(\eta, \xi)=\mathbf{R}^{-1} \mathbf{B}^{-1} \tilde{\mathbf{Q}}^{k l}(\eta, \xi) \mathbf{R}, \quad \overline{\mathbf{L}}_{l}(\xi)=\mathbf{R}^{-1} \mathbf{B}^{-1} \tilde{\boldsymbol{\Gamma}}_{l}(\xi),
$$

$\boldsymbol{\Lambda}$ and $\mathbf{R}$ are the eigenvalue matrix and eigenvector matrix of the determinant $\left(\mathbf{B}^{-1} \mathbf{A}\right)$, respectively. They satisfy the following equality:

$$
\mathbf{B}^{-1} \mathbf{A}=\mathbf{R} \mathbf{\Lambda} \mathbf{R}^{-1}
$$

The solutions of Eq. (22) can be expressed in the form 


$$
\Psi_{l}(\xi)=\left[\begin{array}{cccc}
W_{1}(\xi) & 0 & 0 & 0 \\
0 & W_{2}(\xi) & 0 & 0 \\
0 & 0 & W_{3}(\xi) & 0 \\
0 & 0 & 0 & W_{4}(\xi)
\end{array}\right]\left\{\begin{array}{c}
\sum_{s=0}^{\infty} \bar{A}_{s}^{l} P_{s}^{\left(\alpha_{1}, \beta_{1}\right)}(\xi) \\
\sum_{s=0}^{\infty} \bar{B}_{s}^{l} P_{s}^{\left(\alpha_{2}, \beta_{2}\right)}(\xi) \\
\sum_{s=0}^{\infty} \bar{C}_{s}^{l} P_{s}^{\left(\alpha_{3}, \beta_{3}\right)}(\xi) \\
\sum_{s=0}^{\infty} \bar{D}_{s}^{l} P_{s}^{\left(\alpha_{4}, \beta_{4}\right)}(\xi)
\end{array}\right\}, \quad l=1,2, \cdots, n
$$

where $P_{s}^{\left(\alpha_{j}, \beta_{j}\right)}(j=1,2, \cdots 4)$ are the Jacobi polynomials, and $W_{j}(\xi)=(1-\xi)^{\alpha_{j}}(1+\xi)^{\beta_{j}}$ is the weight function of Jacobi polynomials,

$$
\alpha_{j}=-\frac{1}{2}+\frac{\mathrm{i}}{2 \pi} \ln \frac{1-\mathrm{i} \gamma_{j}}{1+\mathrm{i} \gamma_{j}}, \quad \beta_{j}=-\frac{1}{2}-\frac{\mathrm{i}}{2 \pi} \ln \frac{1-\mathrm{i} \gamma_{j}}{1+\mathrm{i} \gamma_{j}},
$$

with $\gamma_{j}$ being the elements of the eigenvalue matrix $\Lambda$ and $\bar{A}_{s}^{l}, \bar{B}_{s}^{l}, \bar{C}_{s}^{l}$ and $\bar{D}_{s}^{l}$ being the unknown constants to be determined.

By considering the orthogonality relations of the Jacobi polynomials (Gu et al., 2002)

$$
\int_{-1}^{1} W(x) P_{k}^{(\alpha, \beta)}(x) P_{j}^{(\alpha, \beta)}(x) d x= \begin{cases}0, & k \neq j, \\ \theta_{k}^{(\alpha, \beta)}=\frac{2^{(\alpha+\beta+1)} \Gamma(\alpha+k+1) \Gamma(\beta+k+1)}{k !(\alpha+\beta+2 k+1) \Gamma(\alpha+\beta+k+1)}, & k=j,\end{cases}
$$

together with $P_{0}^{(\alpha, \beta)}(x)=1$, it can be concluded that the single-value condition of Eq. (22), which can be expressed as

$$
\int_{-1}^{1} \psi(\eta) d \eta=0
$$

is identically satisfied if $\bar{A}_{0}^{l}=\bar{B}_{0}^{l}=\bar{C}_{0}^{l}=\bar{D}_{0}^{l}=0$.

Substituting Eq. (25) into Eq. (22) and using the following relations (Gu et al., 2002):

$$
\begin{aligned}
\gamma W(\xi) P_{k}^{(\alpha, \beta)}(\xi)+\frac{1}{\pi} \int_{-1}^{1} W(\eta) P_{k}^{(\alpha, \beta)}(\eta) \frac{1}{\eta-\xi} d \eta= \\
\begin{cases}\frac{\left(1+\gamma^{2}\right)^{1 / 2}}{2} P_{k-1}^{(-\alpha,-\beta)}(\xi), & |\xi|<1, \\
-\left(1+\gamma^{2}\right)^{1 / 2}\left[(\xi-1)^{\alpha}(\xi+1)^{\beta} P_{k}^{(\alpha, \beta)}(\xi)+G_{k}^{\infty}(\xi)\right], & |\xi|>1,\end{cases}
\end{aligned}
$$


where $G_{k}^{\infty}(\xi)$ is the principal part of $W(\xi) P_{k}^{(\alpha, \beta)}(\xi)$ at infinity, we obtain the following algebraic equations:

$$
\begin{aligned}
& \sum_{k=1}^{n} \sum_{s=1}^{N}\left[T_{11 \mid m s}^{k l} \bar{A}_{s}^{k}+T_{12 \mid m s}^{k l} \bar{B}_{s}^{k}+T_{13 \mid m s}^{k l} \bar{C}_{s}^{k}+T_{14 \mid m s}^{k l} \bar{D}_{s}^{k}\right]={ }_{m} L_{l}^{e 1}, \\
& \sum_{k=1}^{n} \sum_{s=1}^{N}\left[T_{21 \mid m s}^{k l} \bar{A}_{s}^{k}+T_{22 \mid m s}^{k l} \bar{B}_{s}^{k}+T_{23 \mid m s}^{k l} \bar{C}_{s}^{k}+T_{24 \mid m s}^{k l} \bar{D}_{s}^{k}\right]={ }_{m} L_{l}^{e 2}, \\
& \sum_{k=1}^{n} \sum_{s=1}^{N}\left[T_{31 \mid m s}^{k l} \bar{A}_{s}^{k}+T_{32 \mid m s}^{k l} \bar{B}_{s}^{k}+T_{33 \mid m s}^{k l} \bar{C}_{s}^{k}+T_{34 \mid m s}^{k l} \bar{D}_{s}^{k}\right]={ }_{m} L_{l}^{e 3}, \\
& \sum_{k=1}^{n} \sum_{s=1}^{N}\left[T_{41 \mid m s}^{k l} \bar{A}_{s}^{k}+T_{42 \mid m s}^{k l} \bar{B}_{s}^{k}+T_{43 \mid m s}^{k l} \bar{C}_{s}^{k}+T_{44 \mid m s}^{k l} \bar{D}_{s}^{k}\right]={ }_{m} L_{l}^{e 4},
\end{aligned}
$$

where $m=0,1, \cdots, N-1$, and

$$
\begin{aligned}
& T_{i j \mid m s}^{k l}=\frac{\left(1+\gamma_{i}^{2}\right)^{1 / 2}}{2} \theta_{s-1}^{\left(-\alpha_{i},-\beta_{i}\right)} \delta_{m(s-1)} \delta_{i j} \delta_{k l}+\frac{1}{\pi} \int_{-1}^{1} \int_{-1}^{1} W_{-i}(\xi) P_{m}^{\left(-\alpha_{i},-\beta_{i}\right)}(\xi) \bar{Q}_{i j}^{k l}(\eta, \xi) W_{j}(\eta) P_{s}^{\left(\alpha_{j}, \beta_{j}\right)}(\eta) d \eta d \xi \\
& i, j=1,2, \cdots, 4, \quad l=1,2, \cdots, n
\end{aligned}
$$

with $W_{-j}(\xi)=(1-\xi)^{-\alpha_{j}}(1+\xi)^{-\beta_{j}}$ and $\delta_{i j}$ being the Kronecker Delta function.

After the constants $\bar{A}_{s}^{l}, \bar{B}_{s}^{l}, \bar{C}_{s}^{l}$ and $\bar{D}_{s}^{l}(s=1,2, \cdots N ; l=1,2, \cdots, n)$ have been determined from Eqs. (30), the equivalent field intensity factors (FIFs) (including stress intensity factors (SIFs), electric displacement intensity factor (EDIF) and magnetic induction intensity factor (MIIF)) of the right crack tip of the $l$ th crack can be defined by extending the FIFs of singule crack in piezoelectric bimaterials (Gu et al., 2002) to magnetoelectroelastic bimaterials. They are

$$
\begin{aligned}
\mathbf{K}_{b_{l}}^{e} & =\left[\begin{array}{l}
K_{\mathrm{Ib}_{1}}^{e} \\
K_{\mathrm{Ib}_{1}}^{e} \\
K_{\mathrm{Db}_{1}}^{e} \\
K_{\mathrm{Bb}_{1}}^{e}
\end{array}\right]=\sqrt{b_{l}-a_{l}} \lim _{\xi \rightarrow 1^{+}}\left[\begin{array}{cccc}
(\xi-1)^{-\alpha_{1}} & 0 & 0 & 0 \\
0 & (\xi-1)^{-\alpha_{2}} & 0 & 0 \\
0 & 0 & (\xi-1)^{-\alpha_{3}} & 0 \\
0 & 0 & 0 & (\xi-1)^{-\alpha_{4}}
\end{array}\right] \\
& \times\left[\Lambda \boldsymbol{\Psi}_{l}(\xi)+\frac{1}{\pi} \int_{-1}^{1} \frac{\boldsymbol{\Psi}_{l}(\eta)}{\eta-\xi} d \eta+\sum_{k=1}^{n} \frac{1}{\pi} \int_{-1}^{1} \overline{\mathbf{Q}}^{k l}(\eta, \xi) \boldsymbol{\Psi}_{k}(\eta) d \eta\right]
\end{aligned}
$$


and the FIFs can be finally expressed as (Gu et al., 2002)

$$
\mathbf{K}_{b_{l}}=\left\{\begin{array}{l}
K_{\mathrm{I} b_{l}} \\
K_{\mathrm{I} b_{l}} \\
K_{\mathrm{D} b_{l}} \\
K_{\mathrm{B} b_{l}}
\end{array}\right\}=-\sqrt{b_{l}-a_{l}} \mathbf{B R} \sum_{s=1}^{N}\left\{\begin{array}{l}
\left(1+\gamma_{1}^{2}\right)^{1 / 2} 2^{\beta_{1}} P_{s}^{\left(\alpha_{1}, \beta_{1}\right)}(1) \bar{A}_{s}^{l} \\
\left(1+\gamma_{2}^{2}\right)^{1 / 2} 2^{\beta_{2}} P_{s}^{\left(\alpha_{2}, \beta_{2}\right)}(1) \bar{B}_{s}^{l} \\
\left(1+\gamma_{3}^{2}\right)^{1 / 2} 2^{\beta_{3}} P_{s}^{\left(\alpha_{3}, \beta_{3}\right)}(1) \bar{C}_{s}^{l} \\
\left(1+\gamma_{4}^{2}\right)^{1 / 2} 2^{\beta_{4}} P_{s}^{\left(\alpha_{4}, \beta_{4}\right)}(1) \bar{D}_{s}^{l}
\end{array}\right\} .
$$

From Eqs. (25), (23) and (14), the extended crack open displacements (CODs) in the vicinity of the right crack tip of the $l$ th crack can be given as

$$
\mathbf{V}_{l}\left(c_{l} \xi+d_{l}\right)=\left\{\begin{array}{c}
\Delta u_{l}\left(c_{l} \xi+d_{l}\right) \\
\boldsymbol{\Delta} w_{l}\left(c_{l} \xi+d_{l}\right) \\
\boldsymbol{\Delta} \phi_{l}\left(c_{l} \xi+d_{l}\right) \\
\Delta \psi_{l}\left(c_{l} \xi+d_{l}\right)
\end{array}\right\}=-c_{l} \mathbf{R}\left\{\begin{array}{l}
\sum_{s=1}^{N} \bar{A}_{s}^{l} \int_{\xi}^{1} W_{1}(\varsigma) P_{s}^{\left(\alpha_{1}, \beta_{1}\right)}(\varsigma) d \varsigma \\
\sum_{s=1}^{N} \bar{B}_{s}^{l} \int_{\xi}^{1} W_{2}(\varsigma) P_{s}^{\left(\alpha_{2}, \beta_{2}\right)}(\varsigma) d \varsigma \\
\sum_{s=1}^{N} \bar{C}_{s}^{l} \int_{\xi}^{1} W_{3}(\varsigma) P_{s}^{\left(\alpha_{3}, \beta_{3}\right)}(\varsigma) d \varsigma \\
\sum_{s=1}^{N} \bar{D}_{s}^{l} \int_{\xi}^{1} W_{4}(\varsigma) P_{s}^{\left(\alpha_{4}, \beta_{4}\right)}(\varsigma) d \varsigma
\end{array}\right\}, \quad \xi \rightarrow 1
$$

where

$$
c_{l}=\frac{b_{l}-a_{l}}{2}, d_{l}=\frac{b_{l}+a_{l}}{2}
$$

Eq. (33) can be further evaluated by

$$
\begin{gathered}
\mathbf{V}_{l}\left(c_{l} \xi+d_{l}\right)=\sqrt{\frac{c_{l}}{2}} \mathbf{R} \operatorname{diag}\left[\frac{(1-\xi)^{\left(1+\alpha_{1}\right)}}{1+\alpha_{1}} \frac{(1-\xi)^{\left(1+\alpha_{2}\right)}}{1+\alpha_{2}} \frac{(1-\xi)^{\left(1+\alpha_{3}\right)}}{1+\alpha_{3}} \frac{(1-\xi)^{\left(1+\alpha_{4}\right)}}{1+\alpha_{4}}\right] \\
\operatorname{diag}\left[\frac{1}{\left(1+\gamma_{1}^{2}\right)^{1 / 2}} \frac{1}{\left(1+\gamma_{2}^{2}\right)^{1 / 2}} \frac{1}{\left(1+\gamma_{3}^{2}\right)^{1 / 2}} \frac{1}{\left(1+\gamma_{4}^{2}\right)^{1 / 2}}\right] \mathbf{K}_{b_{l}}^{e}, \quad \xi \rightarrow 1 .
\end{gathered}
$$

It should be remarked that both the FIFs and CODs in the vicinity of the left crack tip of the $l$ th crack, which are omitted here, can be similarly derived. It is well known that, for magnetoelectrically impermeable cracks, the energy release rates (ERRs) are very important to evaluate the behaviors of crack tips. In accordance with the definition of the energy release rates proposed by Pak (1990), the ERRs of the $l$ th crack can be defined as 


$$
G=\lim _{\Delta L \rightarrow 0} \frac{1}{\Delta L} \int_{0}^{\Delta L} \frac{1}{2} \Pi_{l}(x-\Delta L) \cdot \mathrm{V}_{l}(x) d x
$$

where

$$
\boldsymbol{\Pi}_{l}(x)=\left\{\begin{array}{llll}
\sigma_{z z}(x) & \sigma_{x z}(x) & D_{z}(x) & B_{z}(x)
\end{array}\right\}
$$

and the ERRs can be finally derived as (Soh et al., 2000)

$$
G_{\Xi}=\frac{1}{4} \mathbf{K}_{\Xi}^{e \mathrm{~T}} \mathbf{U} \boldsymbol{\Omega} \mathbf{K}_{\Xi}^{e}, \quad \Xi=b_{l}, a_{l} .
$$

In Eq. (39), the elements of the matrices $\boldsymbol{\Omega}$ and $\mathbf{U}$, which are respectively expressed as $\Omega_{i j}$ and $\mathrm{U}_{i j}$, can be written as follows

$$
\begin{aligned}
& \Omega_{i j}=\left(1+\gamma_{i}^{2}\right)^{-1 / 2} \delta_{i j}, i, j=1,2,3,4, \\
& \mathrm{U}_{i j}=\frac{1}{1+\alpha_{j}} \mathrm{X}_{i j} \frac{\Gamma\left(1+\alpha_{i}\right) \Gamma\left(2+\alpha_{j}\right)}{\Gamma\left(3+\alpha_{i}+\alpha_{j}\right)}, \quad i, j=1,2,3,4,
\end{aligned}
$$

and $\mathrm{X}_{i j}$ is the element of the matrix $\mathbf{X}$, which can be express as

$$
\mathbf{X}=\mathbf{R}^{\mathrm{T}} \mathbf{B}^{\mathrm{T}} \mathbf{R} .
$$

It should be noted that Eq. (39) has the same form as the ERR given by Gu et al. (2002) for the interfacial crack problems of piezoelectric bimaterials, which implies that if the piezomagnetic efect and magnetoelectric coupling effect are neglected, Eq. (39) can be deduced to the corresponding results for piezoelectri bimaterials. Moreover, as medium 1 and medium 2 are the same materials, $\alpha_{i} \equiv-\frac{1}{2}(i=1,2,3,4)$, $\frac{\Gamma\left(1+\alpha_{i}\right) \Gamma\left(2+\alpha_{j}\right)}{\Gamma\left(3+\alpha_{i}+\alpha_{j}\right)} \equiv \frac{\pi}{2}$, and $\boldsymbol{\Omega} \equiv \mathbf{I}$ (the $4 \times 4$ identity matrix), thus, the ERR can be expressed as

$$
G_{\Xi}=\frac{\pi}{4} \mathbf{K}_{\Xi}^{\mathrm{T}} \mathbf{B}^{-1} \mathbf{K}_{\Xi},
$$

which is, in fact, the same as those given before (Zhou et al., 2007; Li and Kardomateas, 2007). 


\section{Numerical examples}

In this section, some typical numerical calculations are carried out. The fracture behaviors of one interfacial crack and two interfacial cracks are discussed. For simplicity, in all our numerical procedure, $\tau_{0}$ is assumed to be zero, which implies that only the mode-I interfacial crack problems are investigated in present work. In addition, in our numerical examples, without loss of generality, $\sigma_{0}$ is always taken as $4.2 \times 10^{6} \mathrm{~N} / \mathrm{m}^{2}$.

As a special example, the normalized ERRs of a central crack in a homogeneous magnetoelectroelastic layer versus both electrical and magnetic loads are firstly calculated for various ratios of layer height to half crack length, where magnetoelectroelastic body is taken as $\mathrm{CoFe}_{2} \mathrm{O}_{4}-\mathrm{BaTiO}_{3}$ composite with volume percentage (or volume fraction) $v_{f}=0.2$, the material properties of which are listed in Table 1 (Annigeri et al., 2007). Numerical results are plotted in Fig. 2, where $G_{0}$ represents the ERR of a magnetoelectrically impermeable crack in an infinite magnetoelectroelastic solid; $a$ is the half crack length. $\lambda_{D}=D_{0} e_{15}^{(1)} /\left(\sigma_{0} \varepsilon_{11}^{(1)}\right)$ and $\lambda_{B}=B_{0} f_{15}^{(1)} /\left(\sigma_{0} \mu_{11}^{(1)}\right)$ are the introduced loading combination parameters, which are used to reflect the loading combinations between electrical and mechanical loads, and between magnetic and mechanical loads, respectively. It should be pointed out that in our numerical procedures (including what follows), trial $N$ is taken as 10. Fig. 2 shows that whether the increasing or decreasing of the ERRs depends on not only the amplitudes but also the directions of the applied electrical load and/or magnetic load. Fig. 2 also indicates that for a central crack in a homogeneous magnetoelectroelastic body, the magnetic load has much smaller influence on the ERRs than the electrical load. In addition, it should be noted that as $\lambda_{D}=\lambda_{B}=0$, the normalized ERRs 
approach to 1 with the increasing of $h_{1} / a=h_{2} / a$. This means that under purely mechanical load, with the increasing of the layer height, the ERRs tend to the one of a central crack situated in an infinite magnetoelectroelastic solid. Thus, to a certain extent, the results have validated our theory.

As an application, the effects of both electrical and magnetic loads on the fracture behaviors of an interfacial crack between two dissimilar $\mathrm{CoFe}_{2} \mathrm{O}_{4}-\mathrm{BaTiO}_{3}$ composites are then examined in this study. Medium 1 and medium 2 correspond to $\mathrm{CoFe}_{2} \mathrm{O}_{4}-\mathrm{BaTiO}_{3}$ composites as $\mathrm{v}_{\mathrm{f}}=0.2$ and as $\mathrm{v}_{\mathrm{f}}=0.4$, respectively. The material constants of medium 2 (i.e., material properties of $\mathrm{CoFe}_{2} \mathrm{O}_{4}-\mathrm{BaTiO}_{3}$ as $\mathrm{v}_{\mathrm{f}}=0.4$ ) are simultaneously listed in Table 1 . Fig. 3 shows that similar to a central crack situated in a homogeneous magnetoelectroelastic layer, for a small $\left|\lambda_{D}\right|$ and $\left|\lambda_{B}\right|$, at least for the material combinations considered here, both negative $\lambda_{D}$ and $\lambda_{B}$ impede interfacial crack to propagate and grow, and both positive $\lambda_{D}$ and $\lambda_{B}$ enhance the crack propagation and growth, and that the electrical load has much bigger effects on the ERRs than the magnetic load. Comparing Fig. 3 with Fig. 2, it is also seen that as $h_{1} / a=h_{2} / a$, for a fixed $h_{1} / a$, the normalized ERRs of an interfacial crack are always larger than the corresponding ones of a central crack in a homogeneous material. It should be pointed out that $G_{0}$ in Fig. 3 (and in what follows) has the same meaning as the one presented in Fig. 2, i.e., $G_{0}$ represents the energy release rate for infinite medium 1 containing a magnetoelectrically impermeable crack of length $2 a$ under purely mechanical load.

The effects of material properties on the normalized ERRs under a pure mechanical load are further examined in this section. Medium 1 is still taken as $\mathrm{CoFe}_{2} \mathrm{O}_{4}-\mathrm{BaTiO}_{3}$ composite with $v_{f}=0.2$. The material properties of medium 2 are 
determined by the ratios defined as follows:

$$
\begin{aligned}
& c_{11}^{(2)} / c_{11}^{(1)}=r_{1}, c_{13}^{(2)} / c_{13}^{(1)}=r_{2}, c_{33}^{(2)} / c_{33}^{(1)}=r_{3}, c_{44}^{(2)} / c_{44}^{(1)}=r_{4}, e_{13}^{(2)} / e_{13}^{(1)}=r_{5}, e_{33}^{(2)} / e_{33}^{(1)}=r_{6}, e_{15}^{(2)} / e_{15}^{(1)}=r_{7}, \\
& f_{13}^{(2)} / f_{13}^{(1)}=r_{8}, f_{33}^{(2)} / f_{33}^{(1)}=r_{9}, f_{15}^{(2)} / f_{15}^{(1)}=r_{10}, \varepsilon_{11}^{(2)} / \varepsilon_{11}^{(1)}=r_{11}, \varepsilon_{33}^{(2)} / \varepsilon_{33}^{(1)}=r_{12}, g_{11}^{(2)} / g_{11}^{(1)}=r_{13}, \\
& g_{33}^{(2)} / g_{33}^{(1)}=r_{14}, \mu_{11}^{(2)} / \mu_{11}^{(1)}=r_{15}, \mu_{33}^{(2)} / \mu_{33}^{(1)}=r_{16}, \rho^{(2)} / \rho^{(1)}=r_{17} .
\end{aligned}
$$

Numerical results are plotted in Figs. 4-10, where except for the material parameters pointed out in the corresponding curves, the other material constants remain the same as medium 1. Fig. 4 shows that the normalized ERRs decrease with the increasing of $r_{1}, r_{3}$ and $r_{4}$, and that on the contrary, the normalized ERRs increase with the increasing of $r_{2}$. Fig. 5 indicates that the normalized ERRs decrease with the increasing either of $r_{5}$ or of $r_{6}$. However, the ERRs are nearly independent of $r_{7}$. From Fig. 6, it is easily seen that both decreasing $r_{9}$ and increasing $r_{10}$ can decrease the ERRs, i.e., impede the interfacial crack propagation and growth. Fig. 6 also implies that adjusting $r_{8}$ has relatively smaller effects on the normalized ERRs than adjusting either $r_{9}$ or $r_{10}$. Fig. 7 shows decreasing either $r_{11}$ or $r_{12}$, i.e., decreasing dielectric permittivities can always retard the interfacial crack propagation. Figs. 8-10 display that both increasing $r_{14}$ and decreasing $r_{15}$ and/or $r_{16}$ only inhabit the crack propagation and growth slightly, and that adjusting both $r_{13}$ and $r_{17}$ almost has no influence on the fracture behaviors according to energy release rate criterion.

Finally, the normalized ERRs for the case of two interfacial cracks between two dissimilar magnetoelectroelastic layers are numerically evaluated. As discussed in Fig. 3, medium 1 and medium 2 are also set to be $\mathrm{CoFe}_{2} \mathrm{O}_{4}-\mathrm{BaTiO}_{3}$ composites with $v_{f}=0.2$ and $v_{f}=0.4$, respectively. For briefly, only some numerical results are graphically given in Figs. 11 and 12, where $b_{1}-a_{1}=b_{2}-a_{2}=2 a$, and $G_{0}$ is defined as 
before. As shown in Figs. 11 and 12, for the material combinations considered here, both the electrical and magnetic loads have the same effects as the case of single interfacial crack. As expected, Figs. 11 and 12 also indicate that for a larger $h_{1}\left(=h_{2}\right)$ the distance between the two cracks has insignificant effects on the ERRs.

In addition, it is worth remarking that the ERRs obtained from the numerical procedures in this section are all real. This phenomenon has also been verified for interfacial cracks between two dissimilar magnetoelectroelastic half-planes by Li and Kardomateas (2007), where the extended Stroh's theory and analytic continuation principle of complex analysis have been used.

\section{Conclusions}

In this paper, fracture analyses of interfacial cracks between two dissimilar magnetoelectroelastic layers are investigated. The magnetoelectrically impermeable crack surface condition is adopted. Fourier transform and dislocation density functions are applied to reduce the mixed boundary value problem to a system of Cauchy singular equations, which can be numerically solved. According to energy release rate criterion, the following conclusions may be drawn:

(1) Although the FIFs exhibit oscillation singularity for mode-I interfacial crack problem considered here, the ERRs at least for the present material combinations are always real. Thus, this kind of oscillation singularity does not appear in the ERRs.

(2) For interfacial crack problems, both the amplitudes and directions of the applied magnetoelectrical loads have effects on the crack extension force. At least for the present material combinations, it is easier to inhibit the crack propagation and growth by adjusting electrical load than by adjusting magnetic load.

(3) For a fixed crack length, increasing the heights of magnetoelectroelastic layers 
can impede the interfacial crack initiation and propagation.

(4) Material combinations have important and different effects on the ERRs of interfacial cracks. On one hand, increasing the ratios of material combination parameters $r_{1}, r_{3}, r_{4}, r_{5}, r_{6}$ and $r_{10}$ may inhibit crack initiation and propagation. On the other hand, decreasing the ratios of any one of $r_{2}, r_{9}, r_{11}$ and $r_{12}$ may also suppress crack propagation and growth. In addition, material parameter radios $r_{7}, r_{13}$ and $r_{17}$ almost have no effects on the fracture behaviors of the magnetoelectroelastic material combinations with interfacial cracks, and the effects of $r_{8}, r_{14}$ and $r_{16}$ even including the effects of $r_{15}$ on the ERRs are, in fact, insignificant as well.

\section{Acknowledgement}

The work was supported by Natural Science Fund of China (10772123) and Natural Science Fund for Outstanding Younger of Hebei Province (A2009001624), China.

\section{Appendix A}

$\lambda_{j}(j=1,2, \cdots, 8)$ in Eqs. (7) are the roots of the following equation

$$
\operatorname{Det}[\mathbf{D}(s, \lambda)]=0
$$

where the matrix $\mathbf{D}(s, \lambda)$ is given by

$$
\mathbf{D}(s, \lambda)=\left[\begin{array}{cccc}
c_{44} \lambda^{2}-c_{11} s^{2} & \left(c_{13}+c_{44}\right) \lambda(-i s) & \left(e_{13}+e_{15}\right) \lambda(-i s) & \left(f_{13}+f_{15}\right) \lambda(-i s) \\
\left(c_{13}+c_{44}\right) \lambda(-i s) & c_{33} \lambda^{2}-c_{44} s^{2} & e_{33} \lambda^{2}-e_{15} s^{2} & f_{33} \lambda^{2}-f_{15} s^{2} \\
\left(e_{13}+e_{15}\right) \lambda(-i s) & e_{33} \lambda^{2}-e_{15} s^{2} & -\varepsilon_{33} \lambda^{2}+\varepsilon_{11} s^{2} & -g_{33} \lambda^{2}+g_{11} s^{2} \\
\left(f_{13}+f_{15}\right) \lambda(-i s) & f_{33} \lambda^{2}-f_{15} s^{2} & -g_{33} \lambda^{2}+g_{11} s^{2} & -\mu_{33} \lambda^{2}+\mu_{11} s^{2}
\end{array}\right] .
$$

The functions $a_{j}^{(\alpha)}(s), b_{j}^{(\alpha)}(s)$ and $c_{j}^{(\alpha)}(s)(j=1, \cdots \cdots, 8)$ in Eqs. (7) can be obtained 
by

$$
\begin{aligned}
& a_{j}(s)=-\frac{\left|\begin{array}{lll}
d_{11}\left(s, \lambda_{j}\right) & d_{13}\left(s, \lambda_{j}\right) & d_{14}\left(s, \lambda_{j}\right) \\
d_{21}\left(s, \lambda_{j}\right) & d_{23}\left(s, \lambda_{j}\right) & d_{24}\left(s, \lambda_{j}\right) \\
d_{31}\left(s, \lambda_{j}\right) & d_{33}\left(s, \lambda_{j}\right) & d_{34}\left(s, \lambda_{j}\right)
\end{array}\right|}{\left|\begin{array}{lll}
d_{12}\left(s, \lambda_{j}\right) & d_{13}\left(s, \lambda_{j}\right) & d_{14}\left(s, \lambda_{j}\right) \\
d_{22}\left(s, \lambda_{j}\right) & d_{23}\left(s, \lambda_{j}\right) & d_{24}\left(s, \lambda_{j}\right) \\
d_{32}\left(s, \lambda_{j}\right) & d_{33}\left(s, \lambda_{j}\right) & d_{34}\left(s, \lambda_{j}\right)
\end{array}\right|} \\
& b_{j}(s)=-\frac{\left|\begin{array}{lll}
d_{12}\left(s, \lambda_{j}\right) & d_{11}\left(s, \lambda_{j}\right) & d_{14}\left(s, \lambda_{j}\right) \\
d_{22}\left(s, \lambda_{j}\right) & d_{21}\left(s, \lambda_{j}\right) & d_{24}\left(s, \lambda_{j}\right) \\
d_{32}\left(s, \lambda_{j}\right) & d_{31}\left(s, \lambda_{j}\right) & d_{34}\left(s, \lambda_{j}\right)
\end{array}\right|}{\left|\begin{array}{lll}
d_{12}\left(s, \lambda_{j}\right) & d_{13}\left(s, \lambda_{j}\right) & d_{14}\left(s, \lambda_{j}\right) \\
d_{22}\left(s, \lambda_{j}\right) & d_{23}\left(s, \lambda_{j}\right) & d_{24}\left(s, \lambda_{j}\right) \\
d_{32}\left(s, \lambda_{j}\right) & d_{33}\left(s, \lambda_{j}\right) & d_{34}\left(s, \lambda_{j}\right)
\end{array}\right|} \\
& c_{j}(s)=-\frac{\left|\begin{array}{lll}
d_{12}\left(s, \lambda_{j}\right) & d_{13}\left(s, \lambda_{j}\right) & d_{11}\left(s, \lambda_{j}\right) \\
d_{22}\left(s, \lambda_{j}\right) & d_{23}\left(s, \lambda_{j}\right) & d_{21}\left(s, \lambda_{j}\right) \\
d_{32}\left(s, \lambda_{j}\right) & d_{33}\left(s, \lambda_{j}\right) & d_{31}\left(s, \lambda_{j}\right)
\end{array}\right|}{\left|\begin{array}{lll}
d_{12}\left(s, \lambda_{j}\right) & d_{13}\left(s, \lambda_{j}\right) & d_{14}\left(s, \lambda_{j}\right) \\
d_{22}\left(s, \lambda_{j}\right) & d_{23}\left(s, \lambda_{j}\right) & d_{24}\left(s, \lambda_{j}\right) \\
d_{32}\left(s, \lambda_{j}\right) & d_{33}\left(s, \lambda_{j}\right) & d_{34}\left(s, \lambda_{j}\right)
\end{array}\right|}
\end{aligned}
$$

where $d_{m n}(s, \lambda)(m=1,2,3$ and $n=1,2,3,4)$ are the components of matrix $\mathbf{D}(s, \lambda)$.

\section{Appendix B}

The components of $\mathbf{H}$ in Eqs. (8) are respectively

$$
\begin{aligned}
& h_{1 j}=\left[c_{13}^{(1)}(-i s)+c_{33}^{(1)} a_{j}^{(1)} \lambda_{j}^{(1)}+e_{33}^{(1)} b_{j}^{(1)} \lambda_{j}^{(1)}+f_{33}^{(1)} c_{j}^{(1)} \lambda_{j}^{(1)}\right] e^{\lambda_{j}^{(1)} h_{1}}, \quad h_{1(j+8)}=0, \\
& h_{2 j}=\left[c_{44}^{(1)} \lambda_{j}^{(1)}+c_{44}^{(1)} a_{j}^{(1)}(-i s)+e_{15}^{(1)} b_{j}^{(1)}(-i s)+f_{15}^{(1)} c_{j}^{(1)}(-i s)\right] e^{\lambda_{j}^{(1)} h_{1}}, \quad h_{2(j+8)}=0, \\
& h_{3 j}=\left[e_{13}^{(1)}(-i s)+e_{33}^{(1)} a_{j}^{(1)} \lambda_{j}^{(1)}-\varepsilon_{33}^{(1)} b_{j}^{(1)} \lambda_{j}^{(1)}-g_{33}^{(1)} c_{j}^{(1)} \lambda_{j}^{(1)}\right] e^{\lambda_{j}^{(1)} h_{1}}, \quad h_{3(j+8)}=0, \\
& h_{4 j}=\left[f_{13}^{(1)}(-i s)+f_{33}^{(1)} a_{j}^{(1)} \lambda_{j}^{(1)}-g_{33}^{(1)} b_{j}^{(1)} \lambda_{j}^{(1)}-\mu_{33}^{(1)} c_{j}^{(1)} \lambda_{j}^{(1)}\right] e^{\lambda_{j}^{(1)} h_{1}}, \quad h_{4(j+8)}=0,
\end{aligned}
$$




$$
\begin{aligned}
& h_{5 j}=0, \quad h_{5(j+8)}=\left[c_{13}^{(2)}(-i s)+c_{33}^{(2)} a_{j}^{(2)} \lambda_{j}^{(2)}+e_{33}^{(2)} b_{j}^{(2)} \lambda_{j}^{(2)}+f_{33}^{(2)} c_{j}^{(2)} \lambda_{j}^{(2)}\right] e^{-\lambda_{j}^{(2)} h_{2}}, \\
& h_{6 j}=0, \quad h_{6(j+8)}=\left[c_{44}^{(2)} \lambda_{j}^{(2)}+c_{44}^{(2)} a_{j}^{(2)}(-i s)+e_{15}^{(2)} b_{j}^{(2)}(-i s)+f_{15}^{(2)} c_{j}^{(2)}(-i s)\right] e^{-\lambda_{j}^{(2)} h_{2}}, \\
& h_{7 j}=0, \quad h_{7(j+8)}=\left[e_{13}^{(2)}(-i s)+e_{33}^{(2)} a_{j}^{(2)} \lambda_{j}^{(2)}-\varepsilon_{33}^{(2)} b_{j}^{(2)} \lambda_{j}^{(2)}-g_{33}^{(2)} c_{j}^{(2)} \lambda_{j}^{(2)}\right] e^{-\lambda_{j}^{(2)} h_{2}}, \\
& h_{8 j}=0, \quad h_{8(j+8)}=\left[f_{13}^{(2)}(-i s)+f_{33}^{(2)} a_{j}^{(2)} \lambda_{j}^{(2)}-g_{33}^{(2)} b_{j}^{(2)} \lambda_{j}^{(2)}-\mu_{33}^{(2)} c_{j}^{(2)} \lambda_{j}^{(2)}\right] e^{-\lambda_{j}^{(2)} h_{2}}, \\
& h_{9 j}=\left[c_{13}^{(1)}(-i s)+c_{33}^{(1)} a_{j}^{(1)} \lambda_{j}^{(1)}+e_{33}^{(1)} b_{j}^{(1)} \lambda_{j}^{(1)}+f_{33}^{(1)} c_{j}^{(1)} \lambda_{j}^{(1)}\right], \\
& h_{9(j+8)}=-\left[c_{13}^{(2)}(-i s)+c_{33}^{(2)} a_{j}^{(2)} \lambda_{j}^{(2)}+e_{33}^{(2)} b_{j}^{(2)} \lambda_{j}^{(2)}+f_{33}^{(2)} c_{j}^{(2)} \lambda_{j}^{(2)}\right], \\
& h_{10 j}=\left[c_{44}^{(1)} \lambda_{j}^{(1)}+c_{44}^{(1)} a_{j}^{(1)}(-i s)+e_{15}^{(1)} b_{j}^{(1)}(-i s)+f_{15}^{(1)} c_{j}^{(1)}(-i s)\right] \\
& h_{10(j+8)}=-\left[c_{44}^{(2)} \lambda_{j}^{(2)}+c_{44}^{(2)} a_{j}^{(2)}(-i s)+e_{15}^{(2)} b_{j}^{(2)}(-i s)+f_{15}^{(2)} c_{j}^{(2)}(-i s)\right] \\
& h_{11 j}=\left[e_{13}^{(1)}(-i s)+e_{33}^{(1)} a_{j}^{(1)} \lambda_{j}^{(1)}-\varepsilon_{33}^{(1)} b_{j}^{(1)} \lambda_{j}^{(1)}-g_{33}^{(1)} c_{j}^{(1)} \lambda_{j}^{(1)}\right], \\
& h_{11(j+8)}=-\left[e_{13}^{(2)}(-i s)+e_{33}^{(2)} a_{j}^{(2)} \lambda_{j}^{(2)}-\varepsilon_{33}^{(2)} b_{j}^{(2)} \lambda_{j}^{(2)}-g_{33}^{(2)} c_{j}^{(2)} \lambda_{j}^{(2)}\right], \\
& h_{12 j}=\left[f_{13}^{(1)}(-i s)+f_{33}^{(1)} a_{j}^{(1)} \lambda_{j}^{(1)}-g_{33}^{(1)} b_{j}^{(1)} \lambda_{j}^{(1)}-\mu_{33}^{(1)} c_{j}^{(1)} \lambda_{j}^{(1)}\right], \\
& h_{12(j+8)}=-\left[f_{13}^{(2)}(-i s)+f_{33}^{(2)} a_{j}^{(2)} \lambda_{j}^{(2)}-g_{33}^{(2)} b_{j}^{(2)} \lambda_{j}^{(2)}-\mu_{33}^{(2)} c_{j}^{(2)} \lambda_{j}^{(2)}\right], \\
& h_{13 j}=1, \quad h_{13(j+8)}=-1, \\
& h_{14 j}=a_{j}^{(1)}, \quad h_{14(j+8)}=-a_{j}^{(2)}, \\
& h_{15 j}=b_{j}^{(1)}, \quad h_{15(j+8)}=-b_{j}^{(2)}, \\
& h_{16 j}=c_{j}^{(1)}, \quad h_{16(j+8)}=-c_{j}^{(2)}, \quad(j=1,2, \cdots \cdots, 8) .
\end{aligned}
$$

The constant matrices A and B in Eq. (17) can be expressed as

$$
\mathbf{A}=\left[\begin{array}{cccc}
0 & M_{12} & M_{13} & M_{14} \\
M_{21} & 0 & 0 & 0 \\
M_{31} & 0 & 0 & 0 \\
M_{41} & 0 & 0 & 0
\end{array}\right], \quad \mathbf{B}=\left[\begin{array}{cccc}
M_{11} & 0 & 0 & 0 \\
0 & M_{22} & M_{23} & M_{24} \\
0 & M_{32} & M_{33} & M_{34} \\
0 & M_{42} & M_{43} & M_{44}
\end{array}\right],
$$

where 


$$
\begin{aligned}
& M_{i j}=\lim _{s \rightarrow \infty}\left[K_{i j}(s)\right], i, j=1,2, \cdots, 4, \\
& K_{11}=i \sum_{j=1}^{8} \frac{h_{10 j} \Delta_{13 j}}{(-i s) \Delta}, \quad K_{12}=\sum_{j=1}^{8} \frac{h_{10 j} \Delta_{14 j}}{(-i s) \Delta}, \quad K_{13}=\sum_{j=1}^{8} \frac{h_{10 j} \Delta_{15 j}}{(-i s) \Delta}, \quad K_{14}=\sum_{j=1}^{8} \frac{h_{10 j} \Delta_{16 j}}{(-i s) \Delta}, \\
& K_{21}=\sum_{j=1}^{8} \frac{h_{9 j} \Delta_{13 j}}{(-i s) \Delta}, \quad K_{22}=i \sum_{j=1}^{8} \frac{h_{9 j} \Delta_{14 j}}{(-i s) \Delta}, \quad K_{23}=i \sum_{j=1}^{8} \frac{h_{9 j} \Delta_{15 j}}{(-i s) \Delta}, \quad K_{24}=i \sum_{j=1}^{8} \frac{h_{9 j} \Delta_{16 j}}{(-i s) \Delta}, \\
& K_{31}=\sum_{j=1}^{8} \frac{h_{11 j} \Delta_{13 j}}{(-i s) \Delta}, \quad K_{32}=i \sum_{j=1}^{8} \frac{h_{11 j} \Delta_{14 j}}{(-i s) \Delta}, \quad K_{33}=i \sum_{j=1}^{8} \frac{h_{11 j} \Delta_{15 j}}{(-i s) \Delta}, \quad K_{34}=i \sum_{j=1}^{8} \frac{h_{11 j} \Delta_{16 j}}{(-i s) \Delta}, \\
& K_{41}=\sum_{j=1}^{8} \frac{h_{12 j} \Delta_{13 j}}{(-i s) \Delta}, \quad K_{42}=i \sum_{j=1}^{8} \frac{h_{12 j} \Delta_{14 j}}{(-i s) \Delta}, \quad K_{43}=i \sum_{j=1}^{8} \frac{h_{12 j} \Delta_{15 j}}{(-i s) \Delta}, \quad K_{44}=i \sum_{j=1}^{8} \frac{h_{12 j} \Delta_{16 j}}{(-i s) \Delta},
\end{aligned}
$$

The function matrix $\mathbf{Q}(v, x)$ in Eq. (17) can be written as

$$
\mathbf{Q}(v, x)=\left[\begin{array}{llll}
Q_{11}(v, x) & Q_{12}(v, x) & Q_{13}(v, x) & Q_{14}(v, x) \\
Q_{21}(v, x) & Q_{22}(v, x) & Q_{23}(v, x) & Q_{24}(v, x) \\
Q_{31}(v, x) & Q_{32}(v, x) & q_{33}(v, x) & Q_{34}(v, x) \\
Q_{41}(v, x) & Q_{42}(v, x) & Q_{43}(v, x) & Q_{44}(v, x)
\end{array}\right],
$$

with

$$
\begin{aligned}
& \mathrm{Q}_{i j}(v, x)=\int_{0}^{\infty}\left[K_{i j}(s)-M_{i j}\right] \sin [s(v-x)] d s, \quad i=j=1, \text { or } i=2,3,4, j=2,3,4, \\
& \mathrm{Q}_{i j}(v, x)=\int_{0}^{\infty}\left[K_{i j}(s)-M_{i j}\right] \cos [s(v-x)] d s, \quad i=1, j=2,3,4, \text { or } i=2,3,4, j=1 .
\end{aligned}
$$

\section{References}

Alshits, V.I., Kircher, H.O.K., Ting, T.C.T., 1995. Angularly inhomegenous piezolelectric piezomagnetic magnetoelastic ansotropiec media. Philos. Mag. Lett. 71, 285-288.

Annigeri, A.R., Ganesan, N., Swarnamani, S., 2007. Free vibration behaviour of multiphase and layered magneto-electro-elastic beam. J. Sound Vib. 299, 44-63.

Chue, C.H., Liu, T.J.C, 2005. Magneto-electro-elastic antiplane analysis of a bimaterial $\mathrm{BaTiO}_{3}-\mathrm{CoFe}_{2} \mathrm{O}_{4}$ composite wedge with an interface crack. Theor. Appl. Frac. Mech. 44, 275-296.

Feng, W.J., Pan, E., Wang, X., 2007. Dynamic fracture analysis of a penny-shaped crack in a magnetoelectroelastic layer. Int. J. Solids Struct. 44, 7955-7974.

Feng, W.J., Su, R.K.L., 2006. Dynamic internal crack problem of a functionally 
graded magneto-electro-elastic strip. Int. J. Solids Struct. 43, 5196-5216.

Feng, W.J., Xue, Y., Zou, Z.Z., 2005. Crack growth of an interface crack between two dissimilar magneto-electro-elastic materials under anti-plane mechanical and in-plane electric magnetic impact. Theor. Appl. Frac. Mech. 43, 376-394.

Gao, C.F., Kessler, H., Balke, H., 2003a. Crack problems in magnetoelectroelastic solids. Part I: Exact solution of a crack. Int. J. Eng. Sci. 41, 969-981.

Gao, C.F., Kessler, H., Balke, H., 2003b. Crack problems in magnetoelectroelastic solids. Part II: General solution of collinear cracks. Int. J. Eng. Sci. 41, 983-994.

Gao, C.F., Tong, P., Zhang, T.Y., 2003c. Interfacial crack problems in magneto-electric solids. Int. J. Eng. Sci. 41, 2105-2121.

Gao, C.F., Tong, P., Zhang, T.Y., 2004. Fracture mechanics for a mode III crack in a magnetoelectroelastic solid. Int. J. Solids Struct. 41, 6613-6629.

Gu, B., Yu, S.W., Feng, X.Q., 2002. Transient response of an insulating crack between dissimilar piezoelectric layers under mechanical and electrical impacts. Arch. Appl. Mech. 72, 615-629.

Harshe, G., Dougherty, J.P., Newnham, R.E., 1993. Theoretical modeling of 3-0/0-3 magnetoelectric composites. Int. J. Appl. Electromag. Mech. 4, 161-171.

Huang, J.H., Chiu, Y.H., Liu, H.K., 1998. Magneto-electro-elastic Eshelby tensors for a piezoelectric-piezomagnetic composite reinforced ellipsoidal inclusions. J. Appl. Phys. 83, 5364-5370.

Huang, J.H., Kuo, W.S., 1997. The analysis of piezoelectric/piezomagnetic composite materials containing an ellipsoidal inhomogeneity. J. Appl. Phys. 81, 1378-1386.

Li, J.Y., 2000. Magnetoelectroelastic multi-inclusion and inhomogeneity problems and their applications in composite materials. Int. J. Eng. Sci. 38, 1993-2011.

Li, J.Y., Dunn, M.L., 1998. Anisotropic coupled field inclusion and inhomogeneity 
problems. Philos. Mag. 77, 1341-1350.

Li, R., Kardomateas, G.A., 2006. The mode III interface crack in piezo-electro-magneto-elastic dissimilar bimaterials. ASME J. Appl. Mech. 73, 220-227.

Li, R., Kardomateas, G.A., 2007. The mixed mode I and II interface crack in piezoelectromagneto-elastic anisotropic bimaterials. ASME J. Appl. Mech. 74, 614-627.

Liu, J.X., Liu, X.L., Zhao, Y.B., 2001. Green’s functions for anisotropic magnetoelectroelastic solids with an elliptical cavity or a crack. Int. J. Eng. Sci. 39, 1405-1418.

Li, X.F., 2005. Dynamic analysis of a cracked magnetoelectroelastic medium under antiplane mechanical and inplane electric magnetic impacts. Int. J. Solids Struct. 42, 3185-3205.

Nan, C.W., 1994. Magnetoelectroelastic effect in composites of piezoelectric and piezomagnetic phases. Phys. Rev. B 50, 6082-6088.

Pak, Y.E., 1990. Crack extension force in a piezoelectric material, ASME J. Appl. Mech. 57, 647-653.

Parton, V.Z., Kudryavtsev, B.A., 1988. Electromagnetoelasticity, Gordon and Breach Science Publishers, New York.

Shen, S.P., Kuang, Z.B., 1998. Wave scattering from an interface crack in laminated anisotropic media. Mech. Res. Commun. 25, 509-517.

Sih, G.C., Jones, R., Song, Z.F., 2003. Piezomagnetic and piezoelectric poling effects on mode I and II crack initiation behavior of magnetoelectroelastic materials. Theor. Appl. Frac. Mech. 40, 161-186.

Soh, A.K., Fang, D.N., Lee, K.L., 2000. Analysis of a bi-piezoelectric ceramic layer 
with an interfacial crack subjected to anti-plane shear and in-plane electric loading. Eur. J. Mech. A/Solids 19, 961-977.

Song, Z.F., Sih, G.C., 2003. Crack initiation behavior in magnetoelectroelastic composite under in-plane deformation. Theor. Appl. Frac. Mech. 39, 189-207.

Su, R.K.L., Feng, W.J., Liu, J.X., Zou, Z.Z., 2003. Transient response of coplanar interfacial cracks between two dissimilar piezoelectric strips under anti-plane mechanical and in-plane electrical impacts. ACTA Mechanica Solida Sinica 16, $300-312$.

Tian, W.Y., Gabbert, U., 2004. Multiple crack interaction problem in magnetoelectroelastic solids. Eur. J. Mech. A/Solids 23, 599-614.

Tian, W.Y., Rajapakse, R.K.N.D., 2005. Fracture analysis of magnetoelectroelastic solids by using path independent integrals. Int. J. Fract. 131, 311-335.

Wang, B.L., Han, J.C., Mai, Y.W., 2006. Mode III fracture of a magnetoelectroelastic layer: exact solution and discussion of the crack face electromagnetic boundary conditions. Int. J. Fract. 139, 27-38.

Wang, B.L., Mai, Y.W., 2007. Applicability of the crack-face electromagnetic boundary conditions for fracture of magnetoelectroelastic materials. Int. J. Solids Struct. 44, 387-398.

Wang, B.L., Sun, Y.G., Zhang, H.Y., 2008. Analysis of a penny-shaped crack in magnetoelectroelastic materials. J. Appl. Phys. 103, 083530-1-8.

Wang, X., Shen, Y.P., 2003. Inclusions of arbitrary shape in magnetoelectroelastic composite materials. Int. J. Eng. Sci. 41, 85-102.

Yong, H.D., Zhou, Y.H., 2007. Transient response of a cracked manetoelectroelastic strip under anti-plane impact. Int. J. Solids Struct. 44, 705-717.

Zhao, M.H., Yang, F., Liu, T., 2006. Analysis of a penny-shaped crack in a 
magneto-electro-elastic medium. Philos. Mag. 86, 4397-4416.

Zhong, X.C., Li, X.F., 2007. T-stress analysis for a Griffith crack in a magnetoelectroelastic solid. Arch. Appl. Mech. 78, 117-125.

Zhou, Z.G., Wang, B., Sun, Y.G., 2004. Two collinear interface cracks in magneto-electro-elastic composites. Int. J. Eng. Sci. 42, 1155-1167.

Zhou, Z.G., Wang, J.Z., Wu, L.Z., 2008. Two collinear Mode-I cracks in piezoelectric/piezomagnetic materials. Struct. Eng. Mech. 29, 55-75.

Zhou, Z.G., Zhang, P.W., Wu, L.Z., 2007. The closed form solution of a Mode-I crack in the piezoelectric/piezomagnetic materials. Int. J. Solids Struct. 44, 419-435.

\section{Figure and Table Captions}

Fig.1. Interfacial cracks between two dissimilar magnetoelectroelastic layers.

Fig.2. Normalized ERRs under different (a) electrical and (b) magnetic loads for a magnetoelectrically impermeable central crack in a homogeneous 
magnetoelectroelastic layer for different layer heights.

Fig.3. Normalized ERRs under different (a) electrical and (b) magnetic loads for a magnetoelectrically impermeable interfacial crack between two dissimilar magnetoelectroelastic layers for different layer heights as $h_{1} / h_{2}=1$.

Fig.4. Normalized ERRs versus $r_{1}\left(r_{2}=\ldots=r_{17}=1\right), r_{2}\left(r_{1}=1, r_{3}=\ldots=r_{17}=1\right), r_{3}\left(r_{1}=r_{2}=1\right.$, $\left.r_{4}=\ldots=r_{17}=1\right)$ and $r_{4}\left(r_{1}=r_{2}=r_{3}=1, r_{5}=\ldots=r_{17}=1\right)$ for single magnetoelectrically impermeable interfacial crack under only mechanical load as $h_{1} / a=h_{2} / a=2.0$.

Fig.5. Normalized ERRs versus $r_{5}\left(r_{1}=\ldots=r_{4}=1, r_{6}=\ldots=r_{17}=1\right), r_{6}\left(r_{1}=\ldots=r_{5}=1, r_{7}=\ldots=\right.$ $\left.r_{17}=1\right)$ and $r_{7}\left(r_{1}=\ldots=r_{6}=1, r_{8}=\ldots=r_{17}=1\right)$ for single magnetoelectrically impermeable interfacial crack under only mechanical load as $h_{1} / a=h_{2} / a=2.0$.

Fig.6. Normalized ERRs versus $r_{8}\left(r_{1}=\ldots=r_{7}=1, r_{9}=\ldots=r_{17}=1\right), r_{9}\left(r_{1}=\ldots=r_{8}=1, r_{10}=\ldots=\right.$ $\left.r_{17}=1\right)$ and $r_{10}\left(r_{1}=\ldots=r_{9}=1, r_{11}=\ldots=r_{17}=1\right)$ for single magnetoelectrically impermeable interfacial crack under only mechanical load as $h_{1} / a=h_{2} / a=2.0$.

Fig.7. Normalized ERRs versus $r_{11}\left(r_{1}=\ldots=r_{10}=1, r_{12}=\ldots=r_{17}=1\right)$ and $r_{12}\left(r_{1}=\ldots=r_{11}=1\right.$, $\mathrm{r}_{13}=\ldots=\mathrm{r}_{17}=1$ ) for single magnetoelectrically impermeable interfacial crack under only mechanical load as $h_{1} / a=h_{2} / a=2.0$.

Fig.8. Normalized ERRs versus $r_{13}\left(r_{1}=\ldots=r_{12}=1, r_{14}=\ldots=r_{17}=1\right)$ and $r_{14}\left(r_{1}=\ldots=r_{13}=1\right.$, $\mathrm{r}_{15}=\mathrm{r}_{16}=\mathrm{r}_{17}=1$ ) for single magnetoelectrically impermeable interfacial crack under only mechanical load as $\mathrm{h}_{1} / \mathrm{a}=\mathrm{h}_{2} / \mathrm{a}=2.0$.

Fig.9. Normalized ERRs versus $r_{15}\left(r_{1}=\ldots=r_{14}=1, r_{16}=r_{17}=1\right)$ and $r_{16} \quad\left(r_{1}=\ldots=r_{15}=1\right.$, $\mathrm{r}_{17}=1$ ) for single magnetoelectrically impermeable interfacial crack under only mechanical load as $h_{1} / a=h_{2} / a=2.0$.

Fig.10. Normalized ERRs versus $r_{17}$ for single magnetoelectrically impermeable interfacial crack under only mechanical load as $\mathrm{h}_{1} / \mathrm{a}=\mathrm{h}_{2} / \mathrm{a}=2.0$ and $r_{1}=r_{2}=\ldots=r_{16}=1$.

Fig.11. Normalized ERRs versus electrical loads at (a) $x=\mathrm{a}_{1}$ and (b) $x=\mathrm{b}_{1}$ for two magnetoelectrically impermeable interface cracks.

Fig.12. Normalized ERRs versus magnetic loads at (a) $x=\mathrm{a}_{1}$ and (b) $x=\mathrm{b}_{1}$ for two magnetoelectrically impermeable interface cracks. 


\section{Table 1}

Material properties of $\mathrm{BaTiO}_{3}-\mathrm{CoFe}_{2} \mathrm{O}_{4}$ composites as a percentage (volume fraction $\left.\mathrm{v}_{\mathrm{f}}\right)\left(c_{\mathrm{ij}}\right.$ in $10^{9} \mathrm{~N} / \mathrm{m}^{2}, e_{\mathrm{ij}}$ in $\mathrm{C} / \mathrm{m}^{2}, \varepsilon_{\mathrm{ij}}$ in $10^{-9} \mathrm{C} / \mathrm{Vm}, f_{\mathrm{ij}}$ in N/Am, $\mu_{\mathrm{ij}}$ in $10^{-4} \mathrm{Ns}^{2} / \mathrm{C}^{2}, g_{\mathrm{ij}}$ in $10^{-12} \mathrm{Ns} / \mathrm{VC}, \rho$ in $\mathrm{kg} / \mathrm{m}^{3}$ ) $\mathrm{v}_{\mathrm{f}}=0.0$ corresponding to $\mathrm{CoFe}_{2} \mathrm{O}_{4}$ and $\mathrm{v}_{\mathrm{f}}=1.0$ to $\mathrm{BaTiO}_{3}$. 


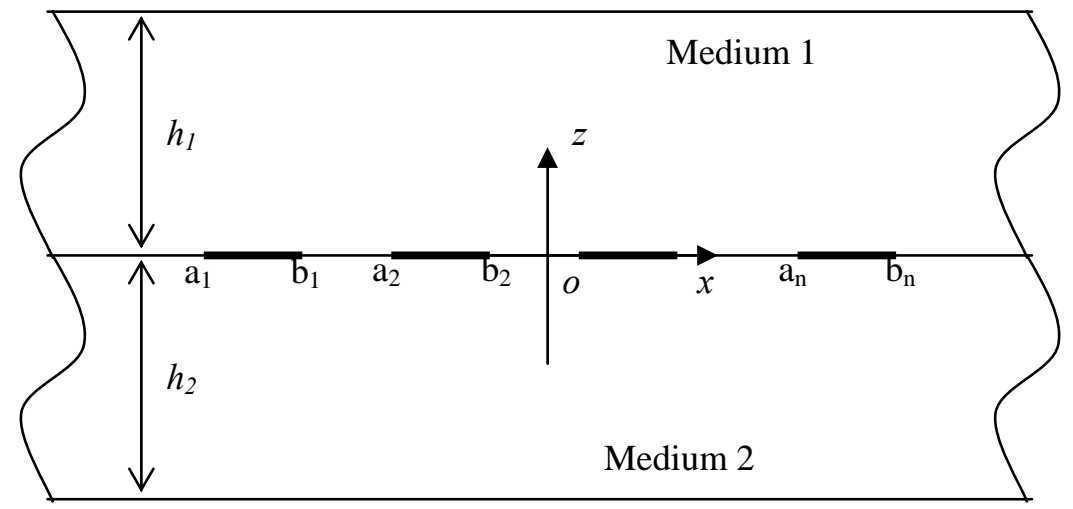

Fig.1. Interfacial cracks between two dissimilar magnetoelectroelastic layers. 
(a)

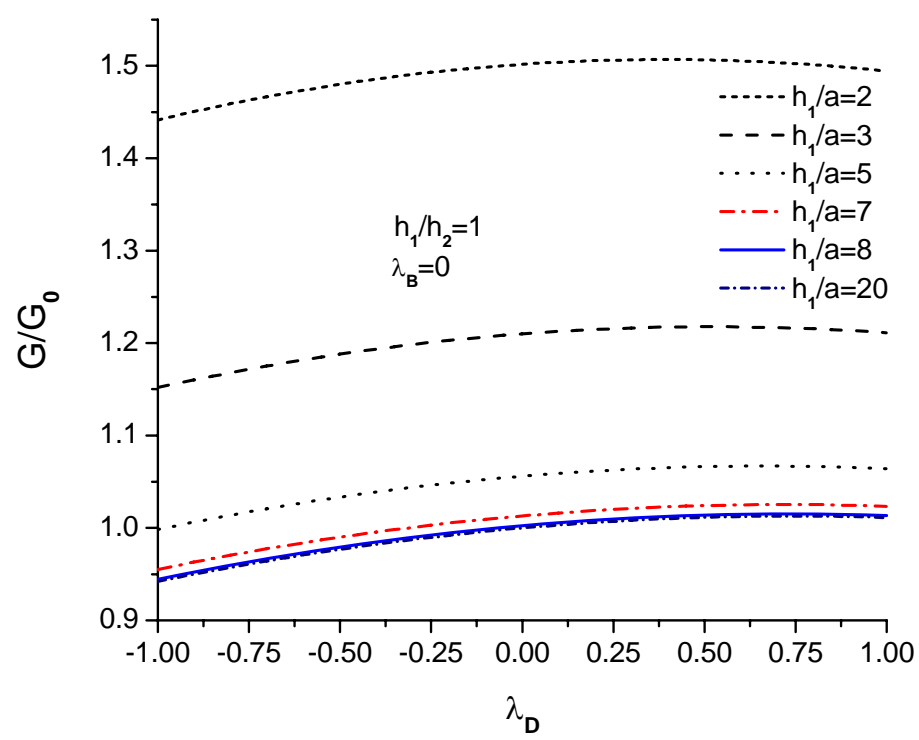

(b)

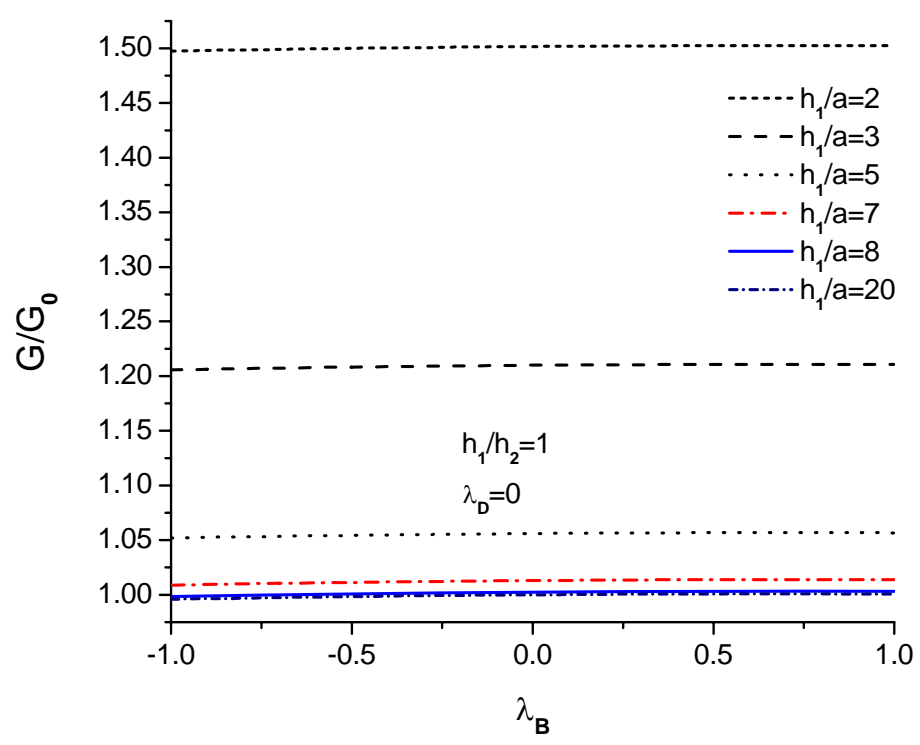

Fig.2. Normalized ERRs under different (a) electrical and (b) magnetic loads for a magnetoelectrically impermeable central crack in a homogeneous magnetoelectroelastic layer for different layer heights. 
(a)

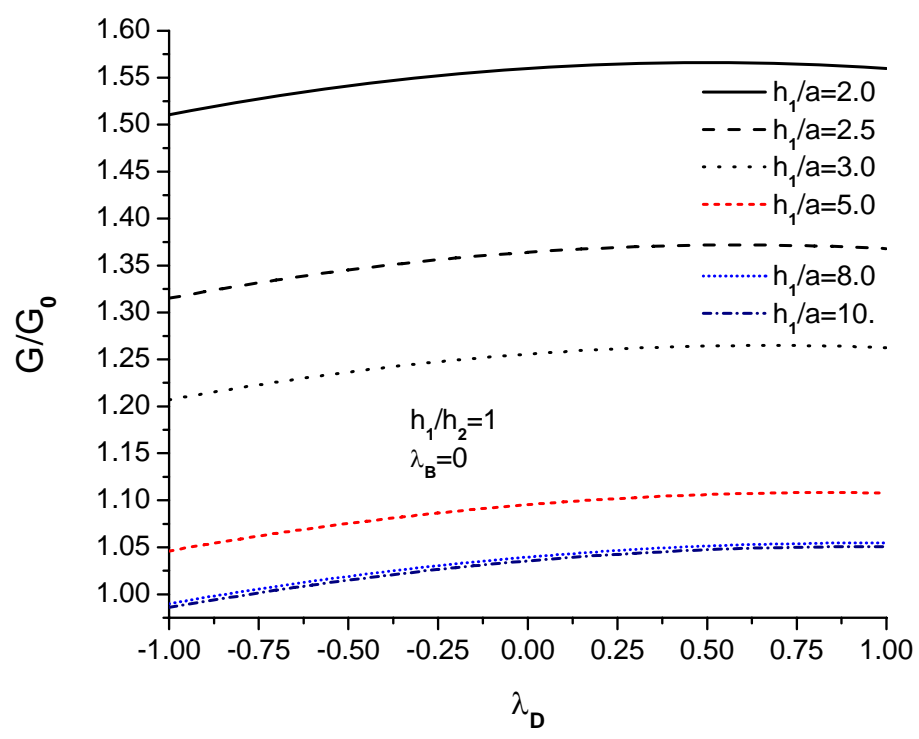

(b)

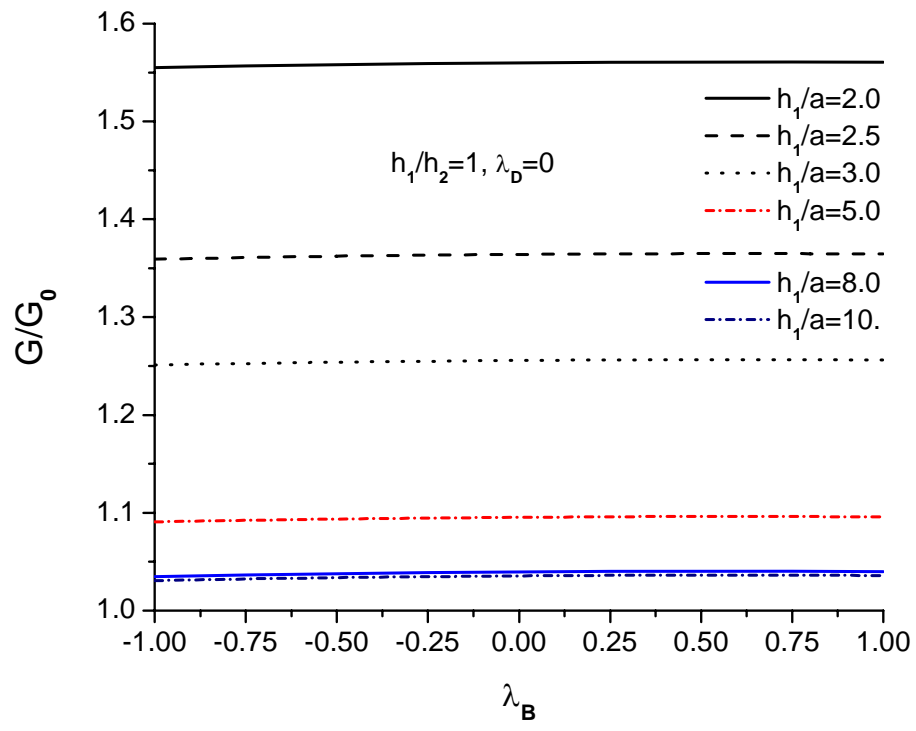

Fig.3. Normalized ERRs under different (a) electrical and (b) magnetic loads for a magnetoelectrically impermeable interfacial crack between two dissimilar magnetoelectroelastic layers for different layer heights as $h_{1} / h_{2}=1$. 


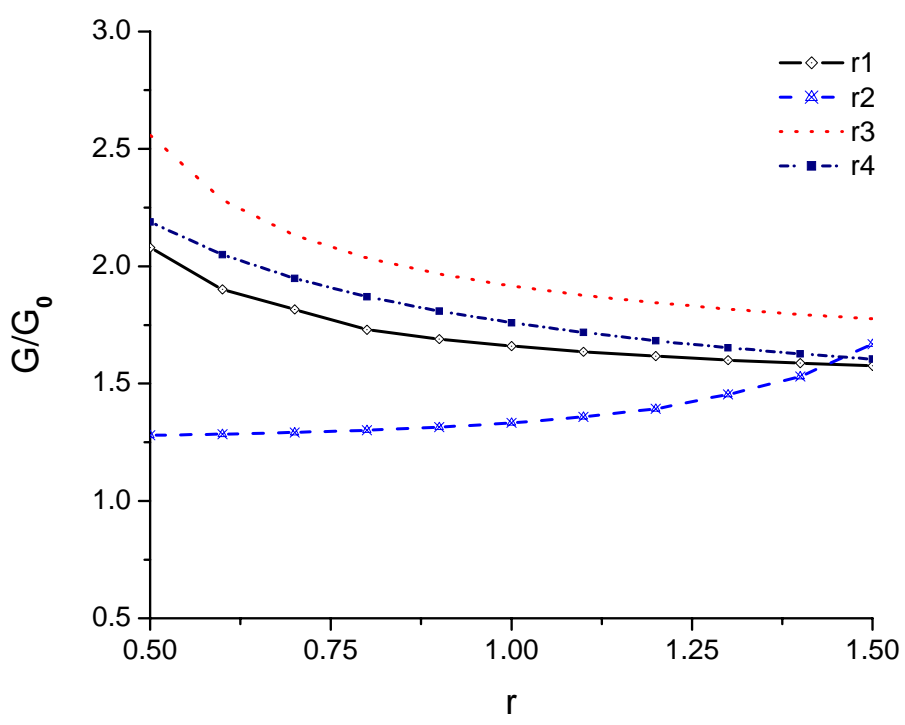

Fig.4. Normalized ERRs versus $r_{1} \quad\left(r_{2}=\ldots=r_{17}=1\right), \quad r_{2} \quad\left(r_{1}=1\right.$, $\left.r_{3}=\ldots=r_{17}=1\right), r_{3}\left(r_{1}=r_{2}=1, r_{4}=\ldots=r_{17}=1\right)$ and $r_{4}\left(r_{1}=r_{2}=r_{3}=1, r_{5}=\ldots=\right.$ $\left.r_{17}=1\right)$ for single magnetoelectrically impermeable interfacial crack under only mechanical load as $h_{1} / a=h_{2} / a=2.0$. 


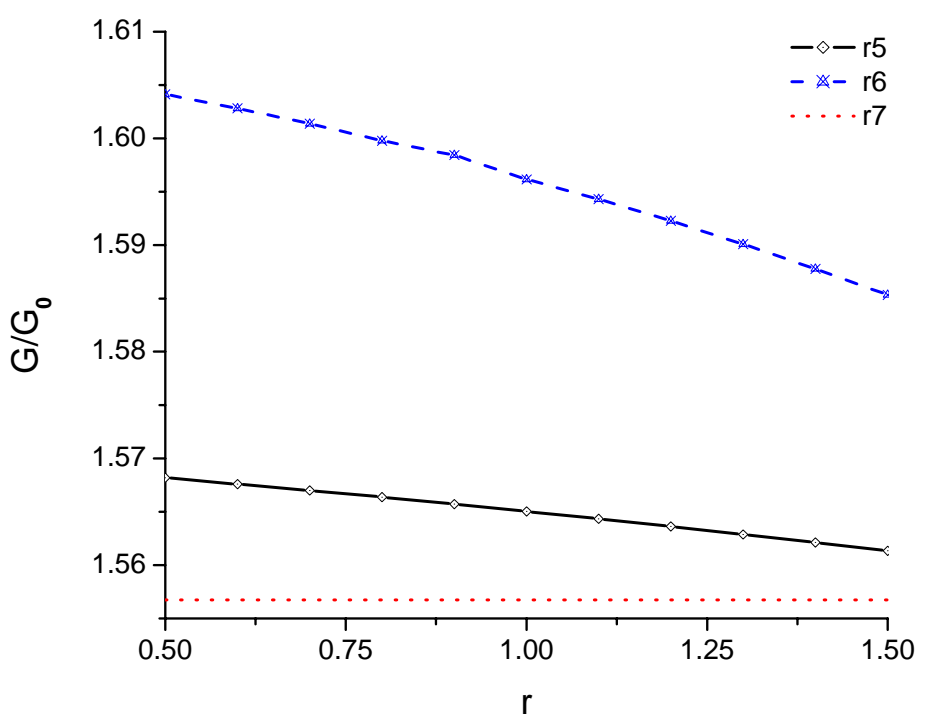

Fig.5. Normalized ERRs versus $r_{5}\left(r_{1}=\ldots=r_{4}=1, r_{6}=\ldots=r_{17}=1\right), r_{6}$ $\left(r_{1}=\ldots=r_{5}=1, r_{7}=\ldots=r_{17}=1\right)$ and $r_{7}\left(r_{1}=\ldots=r_{6}=1, r_{8}=\ldots=r_{17}=1\right)$ for single magnetoelectrically impermeable interfacial crack under only mechanical load as $h_{1} / a=h_{2} / a=2.0$. 


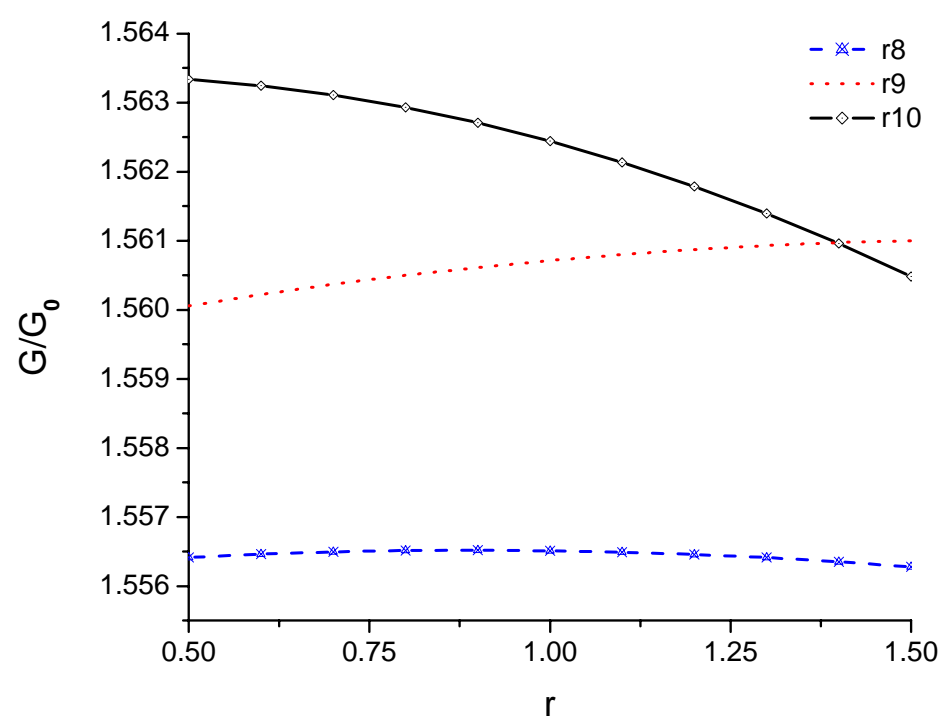

Fig.6. Normalized ERRs versus $r_{8}\left(r_{1}=\ldots=r_{7}=1, r_{9}=\ldots=r_{17}=1\right), r_{9}$ $\left(r_{1}=\ldots=r_{8}=1, r_{10}=\ldots=r_{17}=1\right)$ and $r_{10}\left(r_{1}=\ldots=r_{9}=1, r_{11}=\ldots=r_{17}=1\right)$ for single magnetoelectrically impermeable interfacial crack under only mechanical load as $h_{1} / a=h_{2} / a=2.0$. 


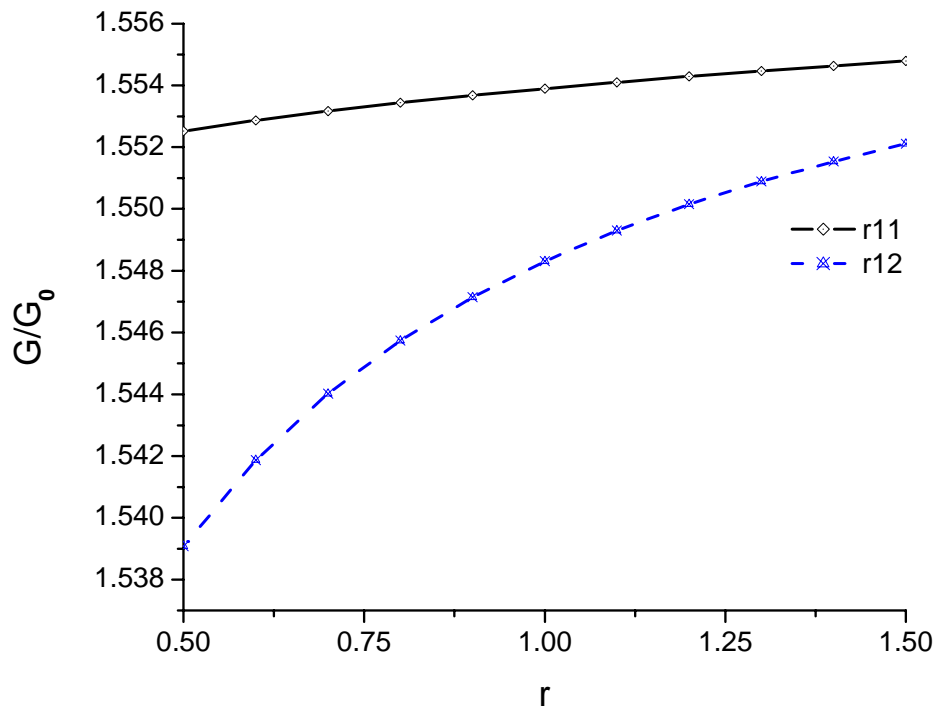

Fig.7. Normalized ERRs versus $r_{11}\left(r_{1}=\ldots=r_{10}=1, r_{12}=\ldots=r_{17}=1\right)$ and $r_{12}$ $\left(r_{1}=\ldots=r_{11}=1, \quad r_{13}=\ldots=r_{17}=1\right)$ for single magnetoelectrically impermeable interfacial crack under only mechanical load as $h_{1} / a=h_{2} / a$ $=2.0$. 


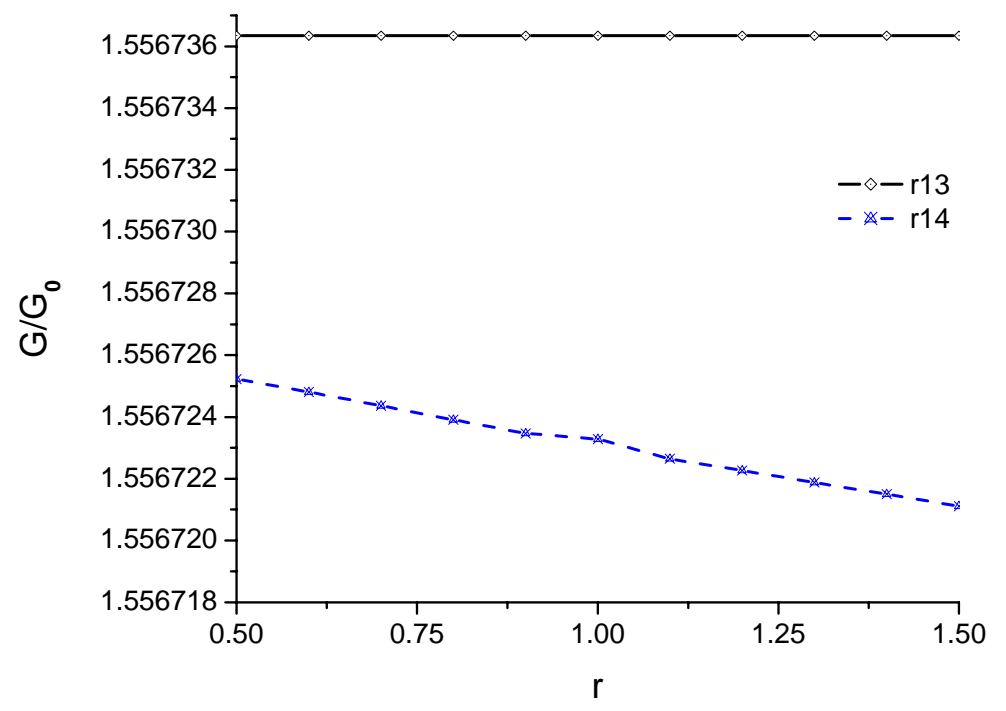

Fig.8. Normalized ERRs versus $r_{13}\left(r_{1}=\ldots=r_{12}=1, r_{14}=\ldots=r_{17}=1\right)$ and $\mathrm{r}_{14} \quad\left(\mathrm{r}_{1}=\ldots=\mathrm{r}_{13}=1, \quad \mathrm{r}_{15}=\mathrm{r}_{16}=\mathrm{r}_{17}=1\right)$ for single magnetoelectrically impermeable interfacial crack under only mechanical load as $h_{1} / a=h_{2} / a$ $=2.0$. 


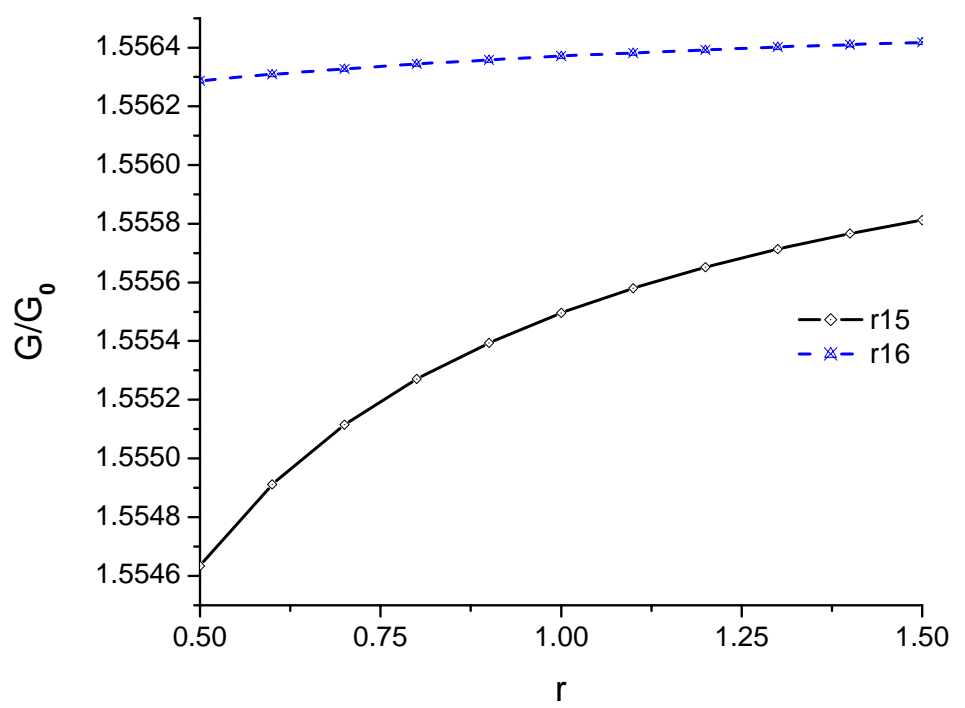

Fig.9. Normalized ERRs versus $r_{15}\left(r_{1}=\ldots=r_{14}=1, r_{16}=r_{17}=1\right)$ and $r_{16}$ $\left(r_{1}=\ldots=r_{15}=1, \quad r_{17}=1\right)$ for single magnetoelectrically impermeable interfacial crack under only mechanical load as $h_{1} / a=h_{2} / a=2.0$. 


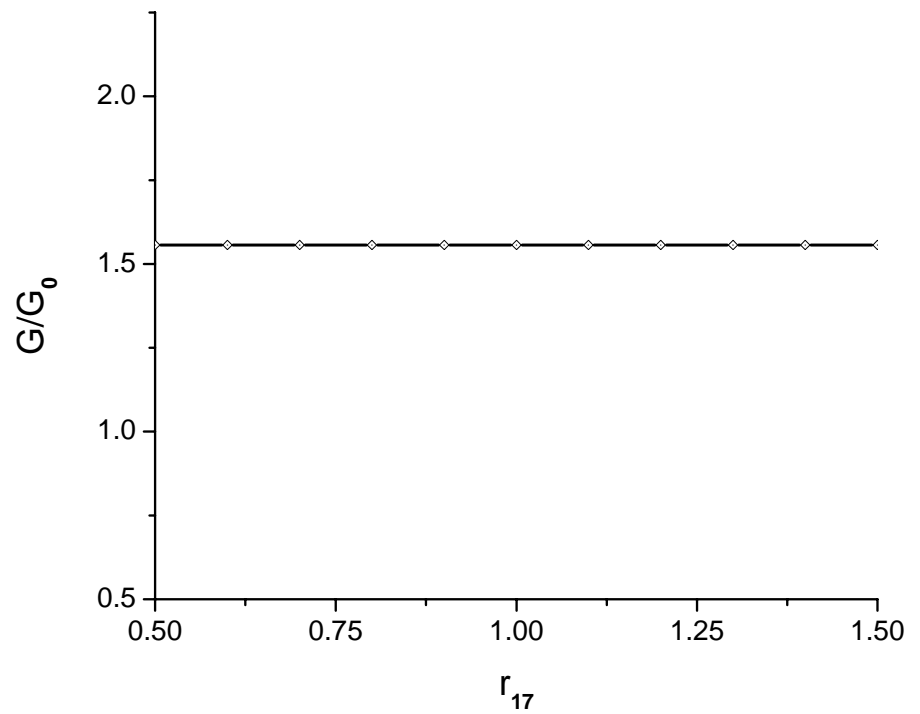

Fig.10. Normalized ERRs versus $r_{17}$ for single magnetoelectrically impermeable interfacial crack under only mechanical load as $h_{1} / a=h_{2} / a$ $=2.0$ and $\mathrm{r}_{1}=\mathrm{r}_{2}=\ldots=\mathrm{r}_{16}=1$. 


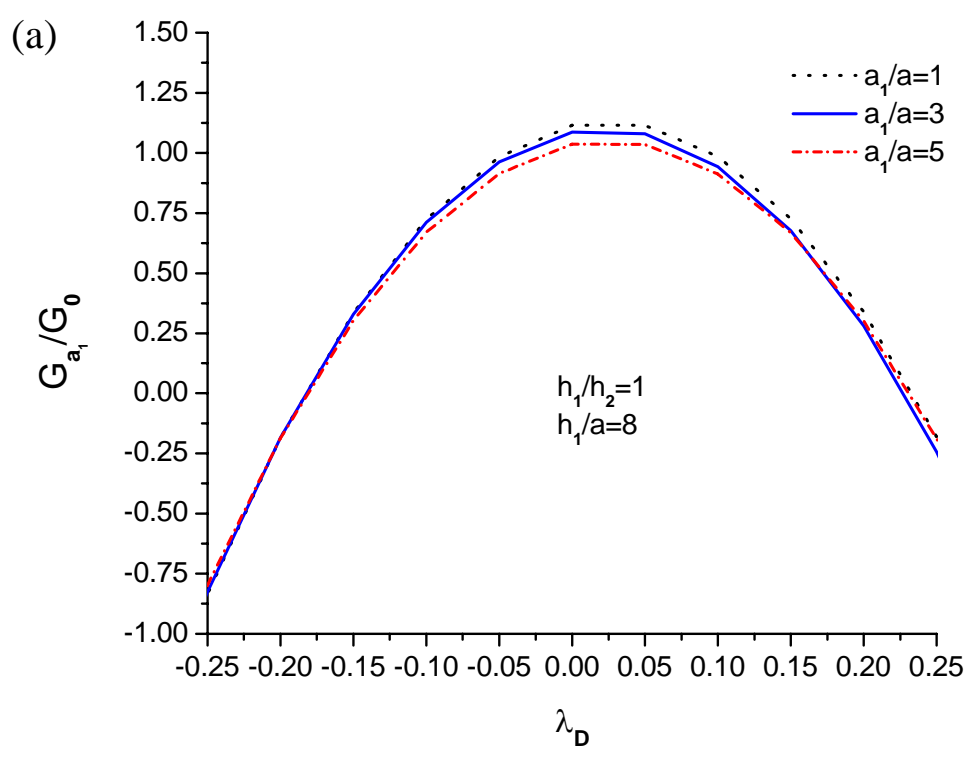

(b)

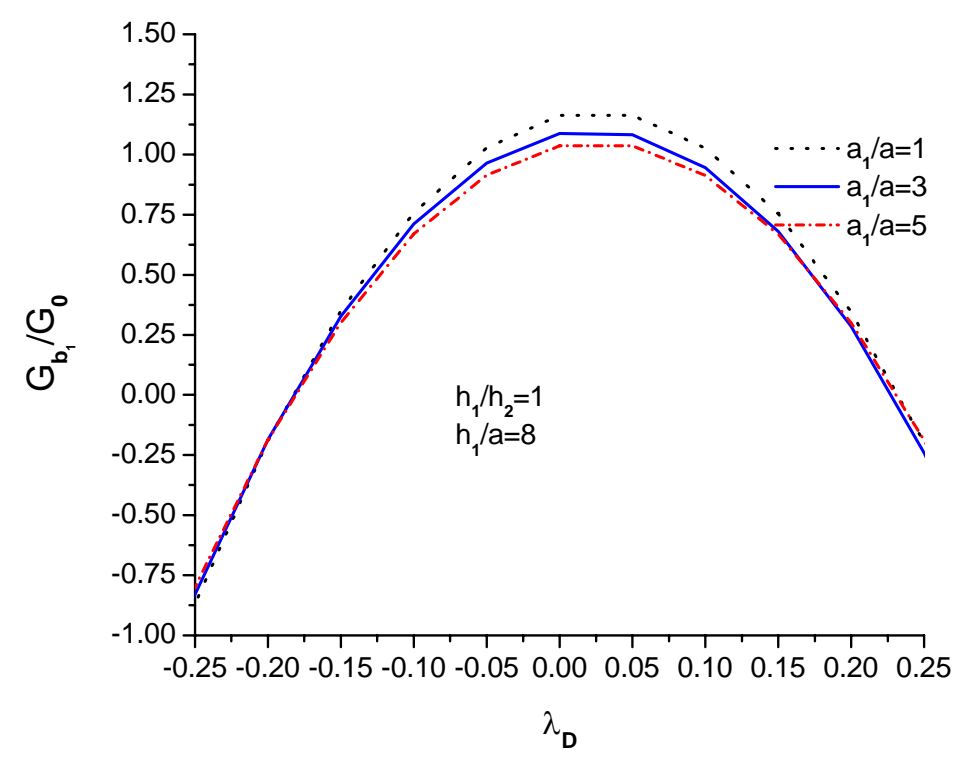

Fig.11. Normalized ERRs versus electrical loads at (a) $x=\mathrm{a}_{1}$ and (b) $x=\mathrm{b}_{1}$ for two magnetoelectrically impermeable interface cracks. 
(a)

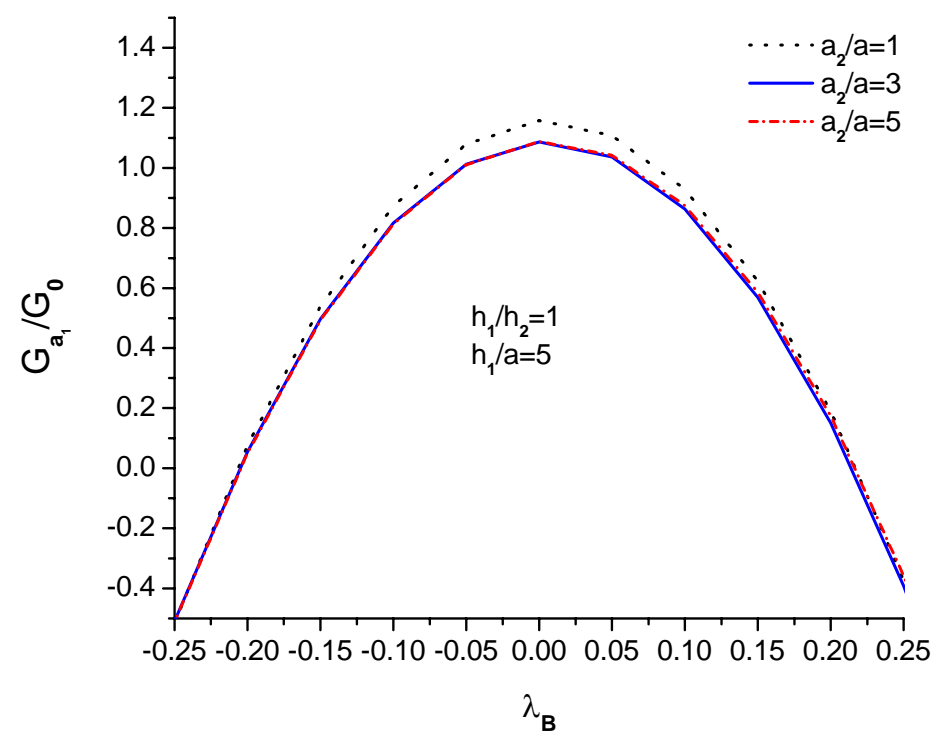

(b)

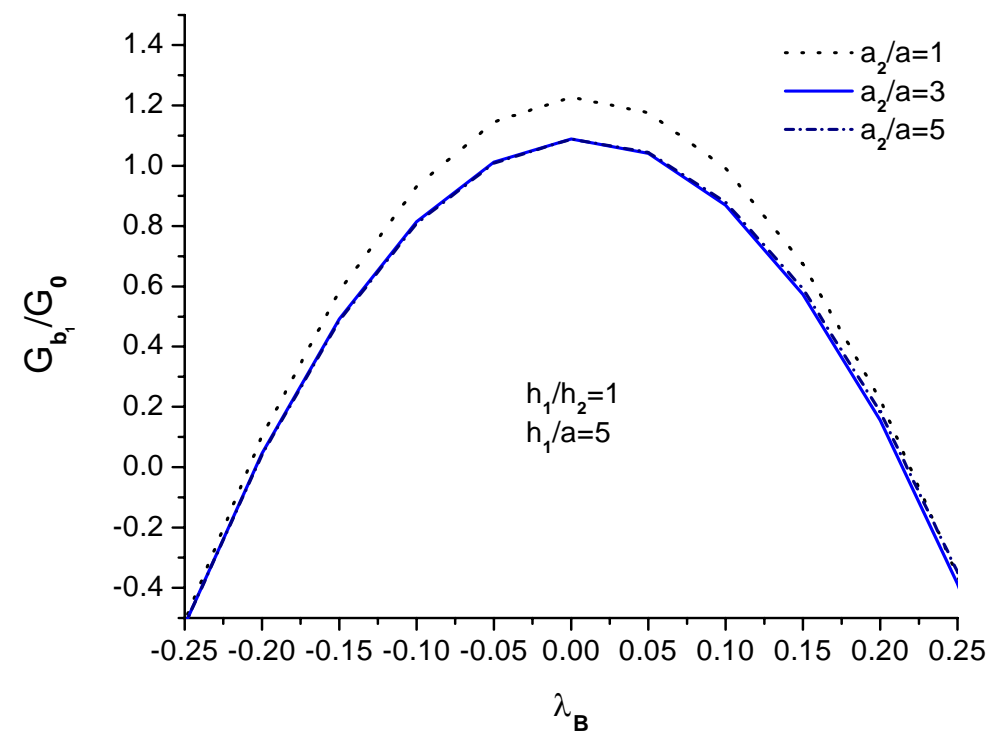

Fig.12. Normalized ERRs versus magnetic loads at (a) $x=\mathrm{a}_{1}$ and (b) $x=\mathrm{b}_{1}$ for two magnetoelectrically impermeable interface cracks. 


\section{Table 1}

Material properties of $\mathrm{BaTiO}_{3}-\mathrm{CoFe}_{2} \mathrm{O}_{4}$ composites as a percentage (volume fraction $\left.\mathrm{v}_{\mathrm{f}}\right)\left(c_{\mathrm{ij}}\right.$ in $10^{9} \mathrm{~N} / \mathrm{m}^{2}, e_{\mathrm{ij}}$ in $\mathrm{C} / \mathrm{m}^{2}, \varepsilon_{\mathrm{ij}}$ in $10^{-9} \mathrm{C} / \mathrm{Vm}, f_{\mathrm{ij}}$ in N/Am, $\mu_{\mathrm{ij}}$ in $10^{-4} \mathrm{Ns}^{2} / \mathrm{C}^{2}, g_{\mathrm{ij}}$ in $10^{-12} \mathrm{Ns} / \mathrm{VC}, \rho$ in $\mathrm{kg} / \mathrm{m}^{3}$ ) $\mathrm{v}_{\mathrm{f}}=0.0$ corresponding to $\mathrm{CoFe}_{2} \mathrm{O}_{4}$ and $\mathrm{v}_{\mathrm{f}}=1.0$ to $\mathrm{BaTiO}_{3}$.

\begin{tabular}{c|ccccccccc}
\hline & $c_{11}$ & $c_{12}$ & $c_{13}$ & $c_{33}$ & $c_{44}$ & $\boldsymbol{e}_{15}$ & $\boldsymbol{e}_{\mathbf{3 1}}$ & $\boldsymbol{e}_{\mathbf{3 3}}$ & $\boldsymbol{\varepsilon}_{\mathbf{1 1}}$ \\
\hline $\mathbf{v}_{\mathbf{f}}=\mathbf{0 . 2}$ & 250 & 146 & 145 & 240 & 45 & 0 & -2 & 4 & 0.33 \\
$\mathbf{v}_{\mathbf{f}}=\mathbf{0 . 4}$ & 225 & 125 & 125 & 220 & 45 & 0 & -3 & 7 & 0.8 \\
\hline & $\boldsymbol{\varepsilon}_{\mathbf{3 3}}$ & $\boldsymbol{\mu}_{\mathbf{1 1}}$ & $\boldsymbol{\mu}_{\mathbf{3 3}}$ & $\boldsymbol{f}_{\mathbf{1 5}}$ & $\boldsymbol{f}_{\mathbf{3 1}}$ & $\boldsymbol{f}_{\mathbf{3 3}}$ & $\boldsymbol{g}_{\mathbf{1 1}}$ & $\boldsymbol{g}_{\mathbf{3 3}}$ & $\boldsymbol{\rho}$ \\
\hline $\mathbf{v}_{\mathbf{f}}=\mathbf{0 . 2}$ & 2.5 & 3.9 & 1.33 & 340 & 410 & 550 & 2.8 & 2000 & 5400 \\
$\mathbf{v}_{\mathbf{f}}=\mathbf{0 . 4}$ & 5.0 & 2.5 & 1.0 & 220 & 300 & 380 & 4.8 & 2750 & 5500 \\
\hline
\end{tabular}

